ESAIM: M2AN 49 (2015) 787-814

DOI: $10.1051 / \mathrm{m} 2 \mathrm{an} / 2014054$
ESAIM: Mathematical Modelling and Numerical Analysis

www.esaim-m2an.org

\title{
REDUCED BASIS TECHNIQUES FOR NONLINEAR CONSERVATION LAWS
}

\author{
T. Taddei ${ }^{1}$, S. Perotto ${ }^{2}$ And A. Quarteroni ${ }^{2,3}$
}

\begin{abstract}
In this paper we present a new reduced basis technique for parametrized nonlinear scalar conservation laws in presence of shocks. The essential ingredients are an efficient algorithm to approximate the shock curve, a procedure to detect the smooth components of the solution at the two sides of the shock, and a suitable interpolation strategy to reconstruct such smooth components during the online stage. The approach we propose is based on some theoretical properties of the solution to the problem. Some numerical examples prove the effectiveness of the proposed strategy.
\end{abstract}

Mathematics Subject Classification. 65M08, 65M15, 65M25, 35L65, 35L67.

Received August 10, 2013. Revised September 29, 2014

Published online 8 April 2015.

\section{INTRODUCTION AND MOTIVATIONS}

The Reduced Basis (RB) method is a Model Order Reduction (MOR) strategy for parametric Partial Differential Equations (PDEs), which is designed to be particularly effective for both a real time and a many-query context.

Given the compact set $\mathcal{D} \subset \mathbb{R}^{P}$ of the parameters and the parametric $\operatorname{PDE} \mathcal{L}(u(\boldsymbol{\mu}), \boldsymbol{\mu})=0$ with $\boldsymbol{\mu} \in \mathcal{D}$, the main goal of the RB approach is to provide a rapid and reliable approximation to the solution manifold $\mathcal{M}:=$ $\{u(\boldsymbol{\mu}): \boldsymbol{\mu} \in \mathcal{D}\}$ through a low dimensional space $W_{N}^{R B}$. The approach is based on three distinct components:

- Rapidly convergent approximations: effective sampling strategies for the construction of the subspaces $\left\{W_{N}^{R B}\right\}_{N}$ are obtained through suitable optimality criteria. Then, a convenient reduced problem, which is efficiently solvable, is introduced.

- Rigorous a posteriori error estimations: the reliability of the reduced solution is assessed through an a posteriori error estimator. The estimate of the RB approximation error has to be obtained via an inexpensive (i.e., independent of the computational mesh), rigorous (i.e., the estimation has to constitute an upper bound for the actual error) and possibly effective (i.e., the ratio of the error bound to the true error is reasonably close to one) way.

\footnotetext{
Keywords and phrases. Nonlinear conservation laws, model reduction, reduced basis method.

1 Department of Mechanical Engineering, Massachusetts Institute of Technology, 77 Massachusetts Ave, Rm. 3-237 Cambridge, MA 02139, USA. ttaddei@mit.edu

2 MOX-Modeling and Scientific Computing, Dipartimento di Matematica "F. Brioschi", Politecnico di Milano, Piazza Leonardo da Vinci 32, 20133 Milano, Italy. simona.perotto@polimi.it; alfio.quarteroni@polimi.it

3 Modelling and Scientific Computing, Institute of Analysis and Scientific Computing, École Polytechnique Féderale de Lausanne, Station 8, EPFL, 1015 Lausanne, Switzerland. alfio.quarteroni@epfl.ch
} 
- Efficient computational offline/online procedures: the global algorithm is divided into two stages: in the former, performed once, the generation of the RB approximation and the computation and storing of all the structures needed for the reduced problem are addressed; in the latter, repeated many times, only the solution of the reduced equation and the error estimation are computed.

Since being first proposed and analyzed in 1970s (see, e.g., [11,25,28]), during the last decade much effort has been devoted to develop this methodology for certain classes of parametric PDEs; rigorous a posteriori error estimation procedures and effective sampling strategies have made the RB method an extremely attractive approach, especially in the case of elliptic and parabolic problems (see, e.g., $[23,27,31]$ ).

This paper is related to the application of the RB method to one dimensional scalar conservation laws depending on a set of parameters, $\boldsymbol{\mu} \in \mathcal{D} \subset \mathbb{R}^{P}$, of the form

$$
\left\{\begin{array}{lc}
\frac{\partial}{\partial t} u(\boldsymbol{\mu})+\frac{\partial}{\partial x} f(u(\boldsymbol{\mu}), \boldsymbol{\mu})=0 & (t, x) \in \Omega_{T,(a, b)} \\
u(\boldsymbol{\mu}, 0, x)=u_{0}(\boldsymbol{\mu}, x) & x \in(a, b)
\end{array}\right.
$$

completed with either periodic or inflow boundary conditions, where $\Omega_{T,(a, b)}=(0, T) \times(a, b)$. The flux $f(\cdot, \boldsymbol{\mu}) \in$ $C^{2}(\mathbb{R})$ is assumed to be uniformly convex (or uniformly concave) in the first argument (i.e., $\left|f^{\prime \prime}(x, \boldsymbol{\mu})\right| \geq \Theta>0$ for any $x \in \mathbb{R}$ and for any $\boldsymbol{\mu} \in \mathcal{D})$, whilst the initial datum $u_{0}(\boldsymbol{\mu}):(a, b) \rightarrow \mathbb{R}$ is assumed to be bounded.

Few papers are available in the literature dealing with MOR strategies for parametric hyperbolic problems: in [7] the so-called Gauss-Newton with approximated tensors (GNAT) method is applied to the parametric inviscid Burgers' equation, whilst in $[9,16]$ the RB method has been suitably extended to linear and nonlinear hyperbolic problems. The main idea in these latter two papers can be thus summarized.

Given $\delta=(\Delta t, h)$, let $\left\{t^{k}=k \Delta t\right\}_{k=0, \ldots, K} \subset[0, T]$ be a time partition of the time window $[0, T]$ and let $X_{h}, Y_{h} \subset L^{2}(a, b)$ be two truth spaces (for instance, the ones induced by a finite element or finite volume discretization) equipped with the inner product $(\cdot, \cdot)_{\star}$. A generic numerical scheme for problem (1.1) can be written in the following form:

$$
\text { find } u_{\delta}(\boldsymbol{\mu})=\left\{u_{\delta}^{k}(\boldsymbol{\mu})\right\}_{k} \in\left[X_{h}\right]^{K+1} \text { such that }\left\{\begin{array}{l}
\left(\mathcal{L}_{\delta}\left(u_{\delta}(\boldsymbol{\mu}), \boldsymbol{\mu}, k\right), v\right)_{\star}=0, \\
\left(u_{h}^{0}(\boldsymbol{\mu})-u_{0}(\boldsymbol{\mu}), v\right)_{\star}=0,
\end{array}\right.
$$

for any $v \in Y_{h}$ and $k=0, \ldots, K$, where $\mathcal{L}_{\delta}$ is the discrete operator induced by the selected numerical scheme. Then, at each time step $t^{k}$, the reduction strategy leads, on the one hand, to project equation (1.2) onto corresponding reduced basis spaces $\left(e . g ., X_{h}^{R B} \subset X_{h}\right.$ and $\left.Y_{h}^{R B} \subset Y_{h}\right)$ and, on the other hand, to approximate the discrete operator $\mathcal{L}_{\delta}$ through a discrete operator $\mathcal{L}_{\delta}^{R B}$, which guarantees an efficient offline/online decomposition.

As we will see in the next section, both the projection of the equation and the interpolation of the discrete operator are particularly problematic when applied to hyperbolic problems. This is the reason why in this work we propose a different approach that, on the one hand, is specifically tailored to an efficient treatment of the shocks and, on the other hand, does not require any approximation of the discrete operator. In more details, first we identify the smooth components of the solution through a domain partition strategy; then, instead of projecting the equation onto a low dimensional subspace, we exploit the method of characteristics (see, e.g., [10]) to efficiently compute the point-wise values of the solution at a certain number of properly selected points. Finally, we reconstruct the entire solution through a suitable interpolation.

Our idea is to some extent related to two MOR techniques that have been proposed recently to deal with nonlinear evolution PDEs. In [13] a MOR strategy based on approximations of Lax-Pairs has been introduced to deal with a certain class of nonlinear problems, while in [26] the so-called "method of freezing" has been applied to decompose the solution into a group and a shape component. As the two above mentioned approaches, our approach is based on the definition of suitable nonlinear approximations to follow the propagating front of the solution, moving from the mathematical analysis of the specific properties of the problem of interest. 
The outline of the paper is as follows. In Section 2 we briefly analyze problem (1.1) and its resolution by reminding some notable results. In Section 3 we introduce the domain partition strategy and we prove a result, extremely useful for the successive interpolation approach. Motivated by this analysis, in Section 4 we introduce the main tools of our method and, finally, in Section 5 some numerical results are presented to assess the proposed strategy. An a posteriori error estimation for the problem at hand is addressed in Appendix A.

\section{The AnAlytical SETting of The PROBlem}

We briefly remind some properties of the solution to problem $(1.1)$. We refer to $[1,10]$ for an exhaustive overview of this topic. For simplicity, we consider problem $(1.1)$ on $\Omega_{T, \infty} \equiv(0, T) \times(-\infty, \infty)$. Throughout the paper, we use standard notation to denote the Lebesgue and Sobolev function spaces and the corresponding norms and seminorms [10].

First, we provide a suitable definition of integral solution to problem (see, e.g., [10]).

Definition 2.1. If $u_{0}(\boldsymbol{\mu}) \in L^{\infty}(\mathbb{R}), u(\boldsymbol{\mu}) \in L^{\infty}\left(\Omega_{T, \infty}\right)$ is said to be a distributional solution to (1.1) if

$$
\int_{\Omega_{T, \infty}}\left(u(\boldsymbol{\mu}) \frac{\partial v}{\partial t}+f(u(\boldsymbol{\mu}), \boldsymbol{\mu}) \frac{\partial v}{\partial x}\right) \mathrm{d} x \mathrm{~d} t+\left.\int_{\mathbb{R}} u_{0}(\boldsymbol{\mu}) v\right|_{t=0} \mathrm{~d} x=0,
$$

for all $v \in C_{0}^{1}((-\infty, T) \times \mathbb{R})$. Furthermore, if $f(\cdot, \boldsymbol{\mu})$ is uniformly convex, $u(\boldsymbol{\mu}) \in L^{\infty}\left(\Omega_{T, \infty}\right)$ is said to be entropic if there exists a constant $C(\boldsymbol{\mu}) \geq 0$ such that the function $u(\boldsymbol{\mu})$ satisfies the inequality:

$$
u(\boldsymbol{\mu}, t, x+z)-u(\boldsymbol{\mu}, t, x) \leq \frac{C(\boldsymbol{\mu})}{t} z
$$

for almost every $x \in \mathbb{R}$ and $t, z \in \mathbb{R}^{+}$. Similarly, if $f(\cdot, \boldsymbol{\mu})$ is uniformly concave, $u(\boldsymbol{\mu}) \in L^{\infty}\left(\Omega_{T, \infty}\right)$ is said to be entropic if there exists a constant $C(\boldsymbol{\mu}) \geq 0$ such that the function $u(\boldsymbol{\mu})$ satisfies the inequality:

$$
u(\boldsymbol{\mu}, t, x-z)-u(\boldsymbol{\mu}, t, x) \leq \frac{C(\boldsymbol{\mu})}{t} z
$$

for almost every $x \in \mathbb{R}$ and $t, z \in \mathbb{R}^{+}$.

Now we introduce two function spaces particularly relevant to study the solution to (2.1). We refer to [2] for further details and comments.

Definition 2.2. Let $\omega \subset \mathbb{R}^{d}$ be an open set and let $u \in L^{1}(\omega)$. Then, $u$ is a function with bounded variation in $\omega$ if the distributional derivative of $u$ can be represented by a finite Radon measure in $\omega$, i.e.,

$$
\int_{\omega} u \frac{\partial \phi}{\partial x_{i}} \mathrm{~d} x=-\int_{\omega} \phi d D_{i} u \quad \forall \phi \in C_{0}^{1}(\omega)
$$

for some $\mathbb{R}^{d}$-valued measure $D u=\left(D_{1} u, \ldots, D_{d} u\right)$ in $\omega$. The vector space of all the functions with bounded variation in $\omega$ is denoted by $B V(\omega)$. Similarly, $u \in B V_{l o c}(\omega)$ if $u \in B V\left(\omega^{\prime}\right)$ for all $\omega^{\prime} \subset \subset \omega$.

Definition 2.3. A function $w \in B V(\omega)$ is a special function with bounded variation, if it can be written as $w=w_{s}+w_{j}$, where $w_{s} \in W^{1,1}(\omega)$ is the smooth part of $w$ while $w_{j}$ is such that $D w_{j}$ is a $(d-1)$ rectifiable measure ${ }^{4}$. The set of the special functions with bounded variation is denoted by $S B V(\omega)$. Similarly, $u \in S B V_{l o c}(\omega)$ if $u \in S B V\left(\omega^{\prime}\right)$ for all $\omega^{\prime} \subset \subset \omega$.

In the sequel, we refer to $w_{s}$ and $w_{j}$ as to the smooth and the jump component of $w$, respectively. Next theorem collects four important results.

\footnotetext{
${ }^{4}$ For more details about the topological structure of the discontinuity set of $w_{j}$, we refer to [2].
} 
Theorem 2.4. Let $u(\boldsymbol{\mu})$ be the entropy solution to problem (2.1).

- Let $x_{s}(\boldsymbol{\mu}):\left(t_{0}, t_{1}\right) \subset(0, T) \rightarrow \mathbb{R}$ be a curve of discontinuity for $u(\boldsymbol{\mu})$ (a so-called shock) and let $u_{\text {left }}(\boldsymbol{\mu}, t)$ and $u_{\text {right }}(\boldsymbol{\mu}, t)$ denote the limit of the integral solution from the left and from the right, respectively. Then, the following identity (known as the Rankine-Hugoniot condition) holds (see, e.g., [10]):

$$
\dot{x}_{s}(\boldsymbol{\mu}, t)=\frac{f\left(u_{\text {right }}(\boldsymbol{\mu}, t), \boldsymbol{\mu}\right)-f\left(u_{\text {left }}(\boldsymbol{\mu}, t), \boldsymbol{\mu}\right)}{u_{\text {right }}(\boldsymbol{\mu}, t)-u_{\text {left }}(\boldsymbol{\mu}, t)} \quad \forall t \in\left(t_{0}, t_{1}\right) .
$$

- Let $f(\cdot, \boldsymbol{\mu}) \in C^{2}(\mathbb{R})$ be a locally uniformly convex function. Then, there exists at most a countable subset $S \subset(0, T)$ such that, for all $\tau \in \mathbb{R} \backslash S$, it holds:

$$
u(\boldsymbol{\mu}, \tau) \in S B V_{l o c}(\mathbb{R}) .
$$

As a consequence $u(\boldsymbol{\mu}) \in S B V_{l o c}\left(\Omega_{T, \infty}\right)[1]$.

- Let $u_{0}(\boldsymbol{\mu}) \in L^{\infty}(\mathbb{R}) \cap B V(\mathbb{R})$, then the following maximum principle holds:

$$
\|u(\boldsymbol{\mu}, t)\|_{L^{\infty}(\mathbb{R})} \leq\left\|u_{0}(\boldsymbol{\mu})\right\|_{L^{\infty}(\mathbb{R})} .
$$

See $[6]$.

- Let $u_{\epsilon}(\boldsymbol{\mu})$ be the entropic distributional solution to the problem:

$$
\left\{\begin{array}{lc}
\frac{\partial}{\partial t} u_{\epsilon}(\boldsymbol{\mu})+\frac{\partial}{\partial x} f_{\epsilon}\left(u_{\epsilon}(\boldsymbol{\mu}), \boldsymbol{\mu}\right)=0(t, x) \in \Omega_{T, \infty} \\
u_{\epsilon}(\boldsymbol{\mu}, 0, x)=u_{0}(\boldsymbol{\mu}, x) & x \in \mathbb{R}
\end{array}\right.
$$

for a suitable flux $f_{\epsilon} \in C^{1}(\mathbb{R})$. Then, the following estimate holds [8]:

$$
\left\|u(\boldsymbol{\mu}, t)-u_{\epsilon}(\boldsymbol{\mu}, t)\right\|_{L^{1}(\mathbb{R})} \leq\left\|u_{0}(\boldsymbol{\mu})\right\|_{B V(\mathbb{R})}\left\|f^{\prime}(\cdot, \boldsymbol{\mu})-f_{\epsilon}^{\prime}(\cdot, \boldsymbol{\mu})\right\|_{L^{\infty}(\mathbb{R})} t \quad \forall t \in(0, T),
$$

where $\left\|u_{0}(\boldsymbol{\mu})\right\|_{B V(\mathbb{R})}=\sup \left\{\int_{\mathbb{R}} u_{0}(\boldsymbol{\mu}) \operatorname{div} \phi \mathrm{d} x: \phi \in C_{0}^{1}(\mathbb{R})\right.$, with $\left.\|\phi\|_{L^{\infty}(\mathbb{R})} \leq 1\right\}$.

Some comments are in order. Condition (2.4) provides a characterization of the shock curve; in addition, by combining (2.4) and (2.6), we easily deduce that each shock curve is Lipschitz-continuous. Moreover, since $x_{s}(\boldsymbol{\mu})$ depends on the solution behaviour in a neighbourhood of this curve, we expect to find a way to compute the shock without knowing the whole solution.

Theorem 2.4 also shows why two crucial components of the classical RB approach (the approximation of the differential operator and the projection onto a Lagrangian subspace of the linear space generated by the parametric manifold) are extremely problematic for the problem at hand. In particular, inequality (2.8) shows that it is crucial to provide a strict approximation of the flux $f(\cdot, \boldsymbol{\mu})$ with respect to the seminorm $|\cdot|_{1, \infty}$, being $|v|_{1, \infty}=\left\|v^{\prime}\right\|_{L^{\infty}(\mathbb{R})}$, to make negligible the difference between the solutions $u(\boldsymbol{\mu})$ and $u_{\epsilon}(\boldsymbol{\mu})$. For this reason, the interpolation of the operator [9] may lead to very long affine expansion of the differential operator; as a consequence, the computational gain that can be achieved online is limited. Concerning the projection step, thanks to (2.5), we can rewrite the solution manifold in the following way:

$$
\mathcal{M}=\left\{u(\boldsymbol{\mu})=u_{s}(\boldsymbol{\mu})+u_{j}(\boldsymbol{\mu}): u_{s}(\boldsymbol{\mu}) \in W_{l o c}^{1,1}\left(\Omega_{T, \infty}\right) \text { and } u_{j}(\boldsymbol{\mu})=\sum_{i=1}^{\infty} \gamma_{i}(\boldsymbol{\mu}) \chi_{\omega_{i}(\boldsymbol{\mu})}\right\},
$$

where $\chi_{\omega}: \Omega_{T, \infty} \rightarrow\{0,1\}$ is the indicator function of the open set $\omega$ and $\left\{\gamma_{i}(\boldsymbol{\mu})\right\}_{i=1}^{\infty} \subset \mathbb{R}$. Thus, due to its intrinsic structure, the manifold $\mathcal{M}$ might not be suited to be approximated through Lagrangian subspaces [27]. The following example is meant to show this issue. 
Example 2.5. Let us consider the manifold

$$
\mathcal{M}_{w}=\left\{w(\mu) \in L^{2}(-5,5): \mu \in[0,1]\right\}, \quad \text { where } w(\mu, x)=\left\{\begin{array}{l}
0 x \leq \mu \\
1 x>\mu
\end{array}\right.
$$

and let $W_{N}=\operatorname{span}\left\{w\left(\mu_{j}\right): \mu_{j} \in[0,1], j=1, \ldots, N\right\}$ be the associated $N$-dimensional Lagrangian approximation space. Then, if $\mu_{j}<\mu_{j+1}$ for all $j$, we have

$$
\inf _{v \in W_{N}}\|w(\mu)-v\|_{L^{2}(-5,5)}=\left\{\begin{array}{l}
\left(\frac{\left(\mu-\mu_{j}\right)\left(\mu_{j+1}-\mu\right)}{\mu_{j+1}-\mu_{j}}\right)^{\frac{1}{2}} \quad \text { if } \exists j \in\{1, \ldots, N-1\}: \mu_{j} \leq \mu \leq \mu_{j+1} \\
\min \left\{\left|\mu-\mu_{1}\right|^{\frac{1}{2}},\left|\mu-\mu_{N}\right|^{\frac{1}{2}}\right\} \text { if } \mu \in\left[0, \mu_{1}\right) \cup\left(\mu_{N}, 1\right] .
\end{array}\right.
$$

In particular, the optimal $N$-dimensional Lagrangian subspace is given by

$$
\begin{aligned}
W_{N}^{\star} & =\arg \inf _{W_{N}}\left(\sup _{\mu \in[0,1]} \inf _{v \in W_{N}}\|w(\mu)-v\|_{L^{2}(-5,5)}\right) \\
& =\operatorname{span}\left\{w\left(\mu_{j}\right): \mu_{j}=\frac{4 j-3}{4 N-2}, j=1, \ldots, N\right\},
\end{aligned}
$$

and

$$
\sup _{\mu \in[0,1]} \inf _{v \in W_{N}^{\star}}\|w(\mu)-v\|_{L^{2}(-5,5)}=\frac{1}{\sqrt{4 N-2}},
$$

i.e., the optimal convergence rate associated with Lagrangian subspaces is linear with respect to $1 / \sqrt{N}$.

We observe that the reduced space $W_{N}$ can be obtained by applying other data compression strategies, such as the Proper Orthogonal Decomposition (POD) (see, e.g., $[15,33]$ ), which has already been applied to non-smooth manifolds [7]. To build the POD basis associated with the manifold (2.10), we define the matrix $C \in \mathbb{R}^{n_{\text {train }} \times n_{\text {train }}}$ such that $C_{i, j}=\frac{1}{n_{\text {train }}}\left(w\left(\mu_{j}\right), w\left(\mu_{i}\right)\right)_{L^{2}(0,1)}$, with $\left\{\mu_{j}\right\}_{j=1}^{n_{\text {train }}} \subset[0,1]$ and $n_{\text {train }} \in \mathbb{N}$. Then, we consider the eigenproblem $C \boldsymbol{\Psi}^{l}=\lambda^{l} \boldsymbol{\Psi}^{l}$ where we assume $\lambda^{l} \geq \lambda^{l+1}, l=1, \ldots, n_{\text {train }}-1$. The $n$-dimensional POD space is thus given by:

$$
X_{n_{\text {train }}}^{n}:=\operatorname{span}\left\{\xi_{l}^{P O D, n_{\text {train }}}=\sum_{i=1}^{n_{\text {train }}} \Psi_{i}^{l} w\left(\mu_{i}\right): l=1, \ldots, n \leq n_{\text {train }}\right\} .
$$

It is straightforward to observe that $X_{n_{\text {train }}}^{n_{\text {train }}}=\operatorname{span}\left\{w\left(\mu_{j}\right)\right\}_{j=1}^{n_{\text {train }}}$. Therefore, because of $(2.12 \mathrm{~b})$, we can deduce that:

$$
\sup _{\mu \in[0,1]} \inf _{v \in X_{n_{\text {train }}}^{n}}\|w(\mu)-v\|_{L^{2}(-5,5)} \geq \frac{1}{\sqrt{4 n_{\text {train }}-2}}, \quad \forall n \in\left\{1, \ldots, n_{\text {train }}\right\} .
$$

As a consequence, the number $n_{\text {train }}$ of snapshots to be selected to guarantee a sufficient accuracy could become extremely large, making the offline computational effort really prohibitive.

\section{A DeCoupling StRategy}

Goal of this section is to provide a new theoretical criterion to tackle hyperbolic problems in the presence of shocks. The leading idea we follow is to properly introduce a partition of the domain induced by the same shock so that the restriction of the solution to each subdomain is regular. 
Let $a, b \in \mathbb{R}$, such that $a<b$. We denote by $u(\boldsymbol{\mu})$ the (unique) entropy solution to the following problem:

$$
\int_{\Omega_{T,(a, b)}}\left(u(\boldsymbol{\mu}) \frac{\partial v}{\partial t}+f(u(\boldsymbol{\mu}), \boldsymbol{\mu}) \frac{\partial v}{\partial x}\right) \mathrm{d} x \mathrm{~d} t+\int_{a}^{b} u_{0}(\boldsymbol{\mu}) v(0, x) \mathrm{d} x=0
$$

for all $v \in C_{0}^{1}((-\infty, T) \times(a, b))$, completed with suitable boundary conditions ${ }^{5}$.

To develop our strategy we need the following three assumptions:

(H1) both $x=a$ and $x=b$ are inflow boundaries for all $t>0$, that is, $f^{\prime}(u(\boldsymbol{\mu}, t, a), \boldsymbol{\mu})>0, f^{\prime}(u(\boldsymbol{\mu}, t, b), \boldsymbol{\mu})<$ 0 [19];

(H2) $u(\boldsymbol{\mu}) \in S B V\left(\Omega_{T,(a, b)}\right)$ and it has only one shock $x_{s}(\boldsymbol{\mu}) \in W^{1, \infty}\left(t^{\star}(\boldsymbol{\mu}), T\right)$ such that $a+\delta \leq x_{s}(\boldsymbol{\mu}, t) \leq b-\delta$ for some $\delta>0$, where $t^{\star}(\boldsymbol{\mu}) \in(0, T)$ denotes the shock starting time;

(H3) we assign the following boundary conditions

$$
u(\boldsymbol{\mu}, t, a)=\xi_{a}(\boldsymbol{\mu}, t), \quad u(\boldsymbol{\mu}, t, b)=\xi_{b}(\boldsymbol{\mu}, t) \quad \forall t \in(0, T)
$$

at $a$ and $b$, respectively, where $\xi_{a}(\boldsymbol{\mu}), \xi_{b}(\boldsymbol{\mu}) \in W^{1,1}(0, T)$. Furthermore, we assume that $u_{0}(\boldsymbol{\mu}) \in W^{1,1}(a, b)$ and that $\xi_{a}(\boldsymbol{\mu}, 0)=u_{0}(\boldsymbol{\mu}, a)$, as well as $\xi_{b}(\boldsymbol{\mu}, 0)=u_{0}(\boldsymbol{\mu}, b)$ to guarantee the continuity of the solution in $(0, a)$ and $(0, b)$. Since $W^{1,1}(I) \subset C(\bar{I})$ with $I \subset \mathbb{R}$ (see, e.g., [32]), we have that all these functions are also continuous up to the boundary.

Thanks to assumptions (H1) and (H2), we can rewrite problem (3.1) as

$$
\left\{\begin{array}{l}
\int_{\Omega_{T,(a, b)}}\left(u(\boldsymbol{\mu}) \frac{\partial v}{\partial t}+f(u(\boldsymbol{\mu}), \boldsymbol{\mu}) \frac{\partial v}{\partial x}\right) \mathrm{d} x \mathrm{~d} t+\int_{a}^{b} u_{0}(\boldsymbol{\mu}) v(0, x) \mathrm{d} x=0, \\
u(\boldsymbol{\mu}, t, a)=\xi_{a}(\boldsymbol{\mu}, t) \quad u(\boldsymbol{\mu}, t, b)=\xi_{b}(\boldsymbol{\mu}, t), \quad \forall t \in(0, T),
\end{array}\right.
$$

for all $v \in C_{0}^{1}((-\infty, T) \times(a, b))$. Now let us consider the following space-time partition induced by the shock $x_{s}(\boldsymbol{\mu})$ (see Fig. 1, left for an example):

$$
\begin{aligned}
& \Omega_{1}(\boldsymbol{\mu})=\left\{(t, x) \in \Omega_{T,(a, b)}: t<t^{\star}(\boldsymbol{\mu})\right\}, \\
& \Omega_{2}(\boldsymbol{\mu})=\left\{(t, x) \in \Omega_{T,(a, b)}: t>t^{\star}(\boldsymbol{\mu}), x<x_{s}(\boldsymbol{\mu}, t)\right\}, \\
& \Omega_{3}(\boldsymbol{\mu})=\left\{(t, x) \in \Omega_{T,(a, b)}: t>t^{\star}(\boldsymbol{\mu}), x>x_{s}(\boldsymbol{\mu}, t)\right\} .
\end{aligned}
$$

We observe that the restriction of the solution $u(\boldsymbol{\mu})$ to each space-time subdomain $\Omega_{i}(\boldsymbol{\mu})$ is regular, i.e., $\left.u(\boldsymbol{\mu})\right|_{\Omega_{i}(\boldsymbol{\mu})} \in W^{1,1}\left(\Omega_{i}(\boldsymbol{\mu})\right)$, for $i=1,2,3$. With a view to the application of the proposed MOR strategy, we aim at referring each solution restriction to a parameter-independent domain. For this purpose, we introduce the maps $\mathbf{T}_{i}(\boldsymbol{\mu}): \Omega_{T,(a, b)} \rightarrow \Omega_{i}(\boldsymbol{\mu})$, for $i=1,2,3$, given by

$$
\begin{aligned}
& \mathbf{T}_{1}(\boldsymbol{\mu}, t, x)=\left[\begin{array}{c}
\frac{t^{\star}(\boldsymbol{\mu})}{T} t \\
T_{x}^{1}(\boldsymbol{\mu}, t, x)
\end{array}\right], \quad \mathbf{T}_{2}(\boldsymbol{\mu}, t, x)=\left[\begin{array}{c}
\tau(\boldsymbol{\mu}, t) \\
a+\frac{x_{s}(\boldsymbol{\mu}, \tau(\boldsymbol{\mu}, t))-a}{b-a}(x-a)
\end{array}\right], \\
& \mathbf{T}_{3}(\boldsymbol{\mu}, t, x)=\left[\begin{array}{c}
\tau(\boldsymbol{\mu}, t) \\
x_{s}(\boldsymbol{\mu}, \tau(\boldsymbol{\mu}, t))+\frac{b-x_{s}(\boldsymbol{\mu}, \tau(\boldsymbol{\mu}, t))}{b-a}(x-a)
\end{array}\right],
\end{aligned}
$$

\footnotetext{
${ }^{5}$ We refer to [3] for a thorough discussion about boundary conditions for first order quasilinear equations.
} 
respectively, with:

$$
T_{x}^{1}(\boldsymbol{\mu}, t, x)=\left\{\begin{array}{lr}
a+\frac{2}{b-a}\left(\gamma\left(\boldsymbol{\mu}, \frac{t^{\star}(\boldsymbol{\mu})}{T} t\right)-a\right)(x-a) & x<\frac{a+b}{2} \\
\gamma(\boldsymbol{\mu}, t)+\frac{2}{b-a}\left(b-\gamma\left(\boldsymbol{\mu}, \frac{t^{\star}(\boldsymbol{\mu})}{T} t\right)\right)\left(x-\frac{a+b}{2}\right) & x \geq \frac{a+b}{2}
\end{array}\right.
$$

where $\tau(\boldsymbol{\mu}, t)=t^{\star}(\boldsymbol{\mu})+\frac{T-t^{\star}(\boldsymbol{\mu})}{T} t$, and

$$
\gamma(\boldsymbol{\mu}, t)= \begin{cases}a+\epsilon+f^{\prime}\left(u_{0}(\boldsymbol{\mu}, a), \boldsymbol{\mu}\right) t & \text { if } a+\epsilon+f^{\prime}\left(u_{0}(\boldsymbol{\mu}, a), \boldsymbol{\mu}\right) t \geq \hat{\gamma}(\boldsymbol{\mu}, t) \\ \hat{\gamma}(\boldsymbol{\mu}, t) & \text { if } a+\epsilon+f^{\prime}\left(u_{0}(\boldsymbol{\mu}, a), \boldsymbol{\mu}\right) t<\hat{\gamma}(\boldsymbol{\mu}, t)<b-\epsilon+f^{\prime}\left(u_{0}(\boldsymbol{\mu}, b), \boldsymbol{\mu}\right) t \\ b-\epsilon+f^{\prime}\left(u_{0}(\boldsymbol{\mu}, b), \boldsymbol{\mu}\right) t & \text { if } b-\epsilon+f^{\prime}\left(u_{0}(\boldsymbol{\mu}, b), \boldsymbol{\mu}\right) t \leq \hat{\gamma}(\boldsymbol{\mu}, t)\end{cases}
$$

being $\hat{\gamma}(\boldsymbol{\mu}, t)=x_{s}\left(\boldsymbol{\mu}, t^{\star}(\boldsymbol{\mu})\right)+\dot{x}_{s}\left(\boldsymbol{\mu}, t^{\star}(\boldsymbol{\mu})\right)\left(t-t^{\star}(\boldsymbol{\mu})\right)$ and $\epsilon>0$. Thanks to hypothesis (H2), the maps $\mathbf{T}_{i}(\boldsymbol{\mu})$ are Lipschitz and bijective for $i=1,2,3$. Then, by construction

$$
u_{i}(\boldsymbol{\mu}):=u(\boldsymbol{\mu}) \circ \mathbf{T}_{i}(\boldsymbol{\mu}) \in W^{1,1}\left(\Omega_{T,(a, b)}\right) \quad \text { for } i=1,2,3 .
$$

Notice that functions $u_{i}(\boldsymbol{\mu})$ correspond to the restrictions $\left.u(\boldsymbol{\mu})\right|_{\Omega_{i}(\boldsymbol{\mu})}$ introduced above even though they are referred to a parameter independent configuration.

We observe that $u_{1}(\boldsymbol{\mu})$ is not continuous up to the boundary. More precisely, it is not continuous in $\left(T, \frac{a+b}{2}\right)$. However, by construction this point is independent of the parameter $\boldsymbol{\mu} \in \mathcal{D}$.

This is why we expect that the convergence of the optimal Lagrangian space associated with the manifold $\left\{u_{1}(\boldsymbol{\mu}): \boldsymbol{\mu} \in \mathcal{D}\right\}$ is enough fast to motivate the proposed reduced order strategy.

On the other hand, hypotheses (H1) and (H3) allow us to introduce the following partition of the space time domain $\Omega_{T,(a, b)}$ :

$$
\left\{\begin{aligned}
R_{1}(\boldsymbol{\mu})= & \left\{(t, x) \in \Omega_{T,(a, b)}: x<a+f^{\prime}\left(u_{0}(\boldsymbol{\mu}, a), \boldsymbol{\mu}\right) t\right\} \cap\left(\Omega_{1}(\boldsymbol{\mu}) \cup \Omega_{2}(\boldsymbol{\mu})\right), \\
R_{2}(\boldsymbol{\mu})= & \left\{(t, x) \in \Omega_{T,(a, b)}: a+f^{\prime}\left(u_{0}(\boldsymbol{\mu}, a), \boldsymbol{\mu}\right) t<x<b+f^{\prime}\left(u_{0}(\boldsymbol{\mu}, b), \boldsymbol{\mu}\right) t, t \leq t^{\star}(\boldsymbol{\mu})\right\}, \\
& \cup\left\{(t, x) \in \Omega_{T,(a, b)}: a+f^{\prime}\left(u_{0}(\boldsymbol{\mu}, a), \boldsymbol{\mu}\right) t<x<\max \left\{x_{s}(\boldsymbol{\mu}, t), b+f^{\prime}\left(u_{0}(\boldsymbol{\mu}, b), \boldsymbol{\mu}\right) t\right\}, t>t^{\star}(\boldsymbol{\mu})\right\}, \\
R_{3}(\boldsymbol{\mu})= & \left\{(t, x) \in \Omega_{T,(a, b)}: x>b+f^{\prime}\left(u_{0}(\boldsymbol{\mu}, b), \boldsymbol{\mu}\right) t\right\} \cap\left(\Omega_{1}(\boldsymbol{\mu}) \cup \Omega_{3}(\boldsymbol{\mu})\right) .
\end{aligned}\right.
$$

Figure 1 shows the difference between the two partitions. While partition $\left\{\Omega_{i}(\boldsymbol{\mu})\right\}_{i=1}^{3}$ is induced by the shock equation and identifies a number of regions where the solution restrictions are regular, partition $\left\{R_{i}(\boldsymbol{\mu})\right\}_{i=1}^{3}$ is induced by the boundary conditions and drives the application of the method of characteristics performed in Proposition 3.1.

We are now ready to state the main result of this paper. We first introduce some notation. We denote by $T_{t}^{i}(\boldsymbol{\mu})$ and $T_{x}^{i}(\boldsymbol{\mu})$ the $t$-component and the $x$-component of the map $\mathbf{T}_{i}(\boldsymbol{\mu})$, respectively, for $i=1,2,3$. Furthermore, we refer to $\mathbf{G}_{i}(\boldsymbol{\mu})$ as to the inverse map of $\mathbf{T}_{i}(\boldsymbol{\mu})$, while $J_{i}(\boldsymbol{\mu}):=\operatorname{det} \nabla \mathbf{T}_{i}(\boldsymbol{\mu})$ denotes the Jacobian of the map $\mathbf{T}_{i}(\boldsymbol{\mu})$, with $i=1,2,3$.

Proposition 3.1. Let us consider the entropy solution $u(\boldsymbol{\mu})$ to (3.1). If the hypotheses (H1)-(H3) are verified, the following statements hold:

- the restrictions $u_{1}(\boldsymbol{\mu}), u_{2}(\boldsymbol{\mu}), u_{3}(\boldsymbol{\mu}) \in W^{1,1}\left(\Omega_{T,(a, b)}\right)$ defined in (3.7) solve the following problems:

$$
\int_{\Omega_{T,(a, b)}}\left(\left[u_{i}(\boldsymbol{\mu}), f\left(u_{i}(\boldsymbol{\mu})\right)\right] \nabla \mathbf{G}_{i}(\boldsymbol{\mu}) \nabla v\right) J_{i}(\boldsymbol{\mu}) \mathrm{d} x \mathrm{~d} t+\left.\left.\int_{a}^{b} u_{0, i}(\boldsymbol{\mu}) J_{i}(\boldsymbol{\mu})\right|_{t=0} v\right|_{t=0} \mathrm{~d} x=0,
$$



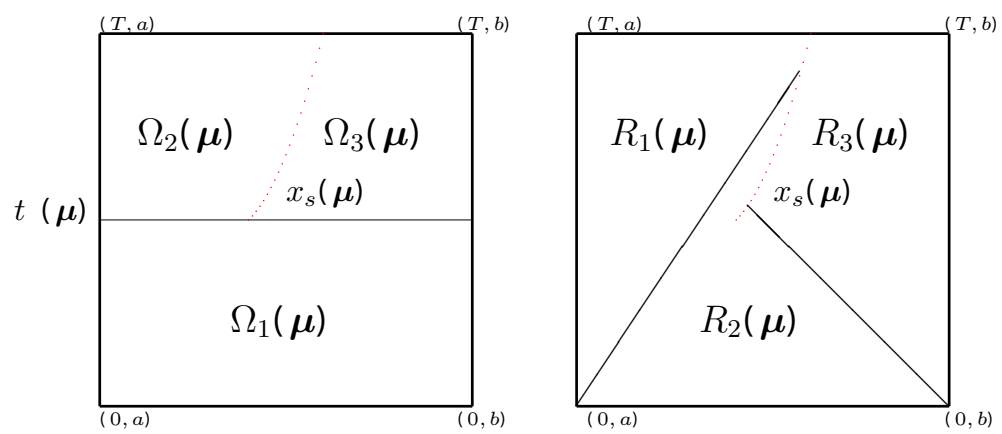

Figure 1. Example of the partitions (3.3) (left) and (3.8) (right); the dotted line (left) represents the shock curve; the continuous lines (right) represent the characteristics issuing from $(0, a)$ and $(0, b)$.

for all $v \in C_{0}^{1}((-\infty, T) \times(a, b))$, where

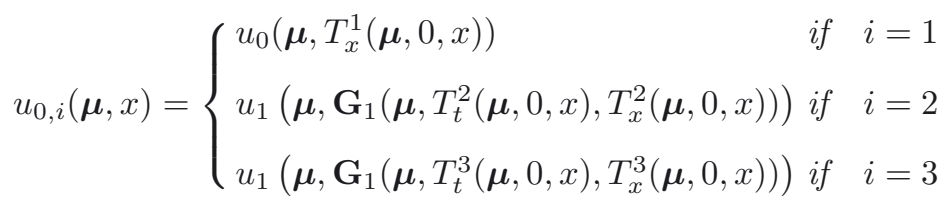

completed with the following boundary conditions:

$$
\begin{aligned}
& u_{1}(\boldsymbol{\mu}, t, a)=\xi_{a}\left(\boldsymbol{\mu}, T_{t}^{1}(\boldsymbol{\mu}, t, a)\right), u_{1}(\boldsymbol{\mu}, t, b)=\xi_{b}\left(\boldsymbol{\mu}, T_{t}^{1}(\boldsymbol{\mu}, t, b)\right) ; \\
& u_{2}(\boldsymbol{\mu}, t, a)=\xi_{a}\left(\boldsymbol{\mu}, T_{t}^{2}(\boldsymbol{\mu}, t, a)\right) ; u_{3}(\boldsymbol{\mu}, t, b)=\xi_{b}\left(\boldsymbol{\mu}, T_{t}^{3}(\boldsymbol{\mu}, t, b)\right),
\end{aligned}
$$

for $t \in(0, T)$;

- if $(t, x) \in R_{i}(\boldsymbol{\mu})$ for some $i=1,2,3$, the pointwise value of the entropy solution $u(\boldsymbol{\mu}, t, x)$ solves the following nonlinear equation:

$$
\left\{\begin{array}{l}
u(\boldsymbol{\mu}, t, x)-\xi_{a}\left(\boldsymbol{\mu}, t-\frac{x-a}{f^{\prime}(u(\boldsymbol{\mu}, t, x), \boldsymbol{\mu})}\right)=0 \text { if }(t, x) \in R_{1}(\boldsymbol{\mu}) \\
u(\boldsymbol{\mu}, t, x)-u_{0}\left(\boldsymbol{\mu}, x-f^{\prime}(u(\boldsymbol{\mu}, t, x), \boldsymbol{\mu}) t\right)=0 \text { if }(t, x) \in R_{2}(\boldsymbol{\mu}) \\
u(\boldsymbol{\mu}, t, x)-\xi_{b}\left(\boldsymbol{\mu}, t-\frac{x-b}{f^{\prime}(u(\boldsymbol{\mu}, t, x), \boldsymbol{\mu})}\right)=0 \text { if }(t, x) \in R_{3}(\boldsymbol{\mu})
\end{array}\right.
$$

Proof. The first statement essentially follows from (3.2) and (3.7) and by applying the change of variable in (3.4)-(3.6).

Thus, let us focus on the second statement of the proposition. We start from region $R_{2}(\boldsymbol{\mu})$. First, we introduce the characteristic lines, which are, generally, defined by

$$
x_{\xi}(\boldsymbol{\mu}, t)=\xi+f^{\prime}\left(u_{0}(\boldsymbol{\mu}, \xi), \boldsymbol{\mu}\right) t,
$$

where $\xi \in(a, b)$ is the so-called foot of the characteristics. Then, we introduce $\xi_{1}=\inf \left\{\xi \in[a, b]: x_{\xi}\left(\boldsymbol{\mu}, t^{\star}(\boldsymbol{\mu})\right)=\right.$ $\left.x_{s}\left(\boldsymbol{\mu}, t^{\star}(\boldsymbol{\mu})\right)\right\}$ and $\xi_{2}=\sup \left\{\xi \in[a, b]: x_{\xi}\left(\boldsymbol{\mu}, t^{\star}(\boldsymbol{\mu})\right)=x_{s}\left(\boldsymbol{\mu}, t^{\star}(\boldsymbol{\mu})\right)\right\}$. Starting from such definitions, we subdivide 


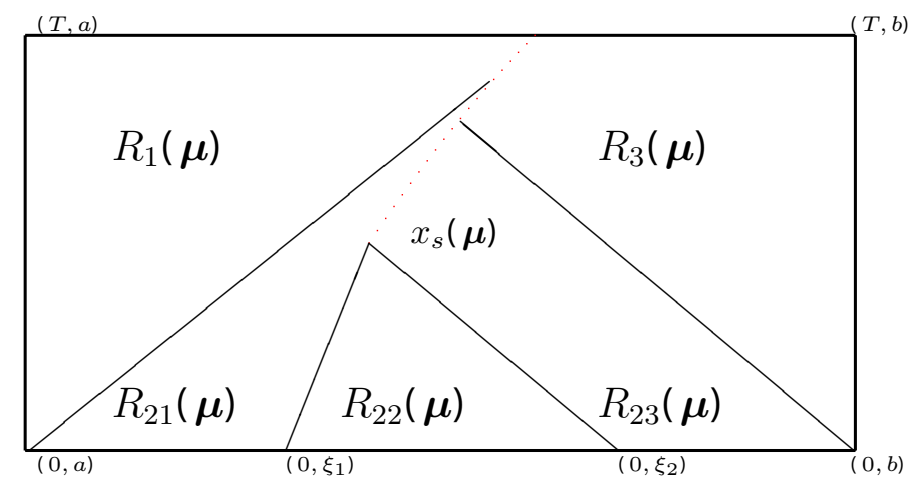

FiguRE 2. Example of partition $\left\{R_{2 i}(\boldsymbol{\mu})\right\}_{i=1}^{3}$. The continuous lines represent the characteristics starting from $a, \xi_{1}, \xi_{2}$ and $b$.

$R_{2}(\boldsymbol{\mu})$ into three subregions, i.e., $R_{21}(\boldsymbol{\mu}), R_{22}(\boldsymbol{\mu}), R_{23}(\boldsymbol{\mu})$, such that

$$
\begin{aligned}
& R_{21}(\boldsymbol{\mu})=\left\{(t, x) \in R_{2}(\boldsymbol{\mu}): x<x_{21}^{\star}(\boldsymbol{\mu}, t)\right\}, \\
& R_{22}(\boldsymbol{\mu})=\left\{(t, x) \in R_{2}(\boldsymbol{\mu}): t<t^{\star}(\boldsymbol{\mu}), x_{\xi_{1}}(\boldsymbol{\mu}, t)<x<x_{\xi_{2}}(\boldsymbol{\mu}, t)\right\}, \\
& R_{23}(\boldsymbol{\mu})=\left\{(t, x) \in R_{2}(\boldsymbol{\mu}): x>x_{23}^{\star}(\boldsymbol{\mu}, t)\right\},
\end{aligned}
$$

with

$$
x_{21}^{\star}(\boldsymbol{\mu}, t)=\left\{\begin{array}{l}
x_{\xi_{1}}(\boldsymbol{\mu}, t) \text { if } t<t^{\star}(\boldsymbol{\mu}) \\
x_{s}(\boldsymbol{\mu}, t) \text { if } t \geq t^{\star}(\boldsymbol{\mu})
\end{array} \quad x_{23}^{\star}(\boldsymbol{\mu}, t)=\left\{\begin{array}{l}
x_{\xi_{2}}(\boldsymbol{\mu}, t) \text { if } t<t^{\star}(\boldsymbol{\mu}) \\
x_{s}(\boldsymbol{\mu}, t) \text { if } t \geq t^{\star}(\boldsymbol{\mu}),
\end{array}\right.\right.
$$

(see Fig. 2 for an example). We observe that $u(\boldsymbol{\mu}) \in W^{1,1}\left(R_{2 i}(\boldsymbol{\mu})\right)$ for $i=1,2,3$.

Let $(\bar{t}, \bar{x}) \in R_{21}(\boldsymbol{\mu})$. In order to prove (3.10), we consider the backward problem

$$
\frac{\partial}{\partial s} u(\boldsymbol{\mu})-\frac{\partial}{\partial x} f(u(\boldsymbol{\mu}), \boldsymbol{\mu})=0
$$

where $s=\bar{t}-t$. If we introduce the characteristic lines for this problem, we obtain:

$$
x_{\bar{x}}^{b a c k}(\boldsymbol{\mu}, s)=\bar{x}-f^{\prime}(u(\boldsymbol{\mu}, \bar{t}, \bar{x}), \boldsymbol{\mu}) s .
$$

For $s>0, x_{\bar{x}}^{b a c k}$ cannot hit the shock as this would violate the Lax entropy condition. Since characteristics do not intersect each other in $R_{21}(\boldsymbol{\mu})$, we finally obtain $x_{\bar{x}}^{\text {back }}(\boldsymbol{\mu}, s) \geq x_{a}(\boldsymbol{\mu}, \bar{t}-s)$, and $x_{\bar{x}}^{b a c k}(\boldsymbol{\mu}, s) \leq x_{21}^{\star}(\boldsymbol{\mu}, \bar{t}-s)$. Therefore, recalling that the solution is constant along the characteristic lines, we have

$$
u(\boldsymbol{\mu}, \bar{t}, \bar{x})=u\left(\boldsymbol{\mu}, \bar{t}, x_{\bar{x}}^{b a c k}(\boldsymbol{\mu}, \bar{t})\right)=u_{0}\left(\boldsymbol{\mu}, \bar{x}-f^{\prime}(u(\boldsymbol{\mu}, \bar{t}, \bar{x}), \boldsymbol{\mu}) \bar{t}\right),
$$

which proves (3.10) for $(t, x) \in R_{21}(\boldsymbol{\mu})$. Subregions $R_{22}(\boldsymbol{\mu})$ and $R_{23}(\boldsymbol{\mu})$ as well as regions $R_{1}(\boldsymbol{\mu})$ and $R_{3}(\boldsymbol{\mu})$ can be dealt in a similar way.

The importance of Proposition 3.1 is twofold. First of all, if we know the shock equation $x_{s}(\boldsymbol{\mu})$, we are able to decouple the starting problem (3.2) into three subproblems whose solutions coincide with the smooth components of $u(\boldsymbol{\mu})$. Moreover, due to the regularity of the manifolds associated with each component, we can approximate these components through a linear combination of a small number of corresponding elements properly selected. 
As a second consequence, equation (3.10) allows us the computation of point-wise values of the entropy solution $u(\boldsymbol{\mu})$ without knowing the whole solution but just via the functions $\xi_{a}$ and $\xi_{b}$ and the initial datum $u_{0}$.

Moving from these remarks, we have settled the decoupling strategy for the shock detection in such a way:

- in the offline stage we compute an approximation $u_{\delta}\left(\boldsymbol{\mu}_{j}\right)$ for $u\left(\boldsymbol{\mu}_{j}\right)$ for some (properly selected) values of the parameter $\boldsymbol{\mu}_{j} \in \mathcal{D}$ with $j=1, \ldots, N$, and for a certain space-time discretization $\delta=(\Delta t, h)$. Then, we approximate $x_{s}\left(\boldsymbol{\mu}_{j}\right)$ and we compute the components $u_{i}\left(\boldsymbol{\mu}_{j}\right)$ via (3.7), i.e., via the maps $\mathbf{T}_{i}\left(\boldsymbol{\mu}_{j}\right)$, for $i=1,2,3$. Now, let

$$
W_{i, N}^{R B}:=\operatorname{span}\left\{u_{i}\left(\boldsymbol{\mu}_{j}\right): j=1, \ldots, N\right\}
$$

denote the reduced space associated with the $i$ th component $u_{i}(\boldsymbol{\mu})$ and with the $N$ parameters $\boldsymbol{\mu}_{j}$. Finally, for each space $W_{i, N}^{R B}$, we define a set of points $\left(t_{i, j}, x_{i, j}\right)$ and a corresponding suitable basis $\left\{q_{i, j}\right\}$ for $j=1, \ldots, N$ and $i=1,2,3$;

- in the online stage, for any new value of the parameter $\boldsymbol{\mu} \in \mathcal{D}$, we first reconstruct the shock $\left\{x_{s}(\boldsymbol{\mu}, t): t \in\right.$ $\left.\left(t^{\star}(\boldsymbol{\mu}), T\right)\right\}$ and then we compute the solution $u(\boldsymbol{\mu})$ in the mapped nodes:

$$
\left(\tilde{t}_{i, j}(\boldsymbol{\mu}), \tilde{x}_{i, j}(\boldsymbol{\mu})\right):=\mathbf{T}_{i}(\boldsymbol{\mu})\left(t_{i, j}, x_{i, j}\right) \quad i=1,2,3, \quad j=1, \ldots, N .
$$

Then, for each $u_{i}(\boldsymbol{\mu})$, we reconstruct a corresponding approximation $u_{i}^{R B}(\boldsymbol{\mu}) \in W_{i, N}^{R B}$ through an interpolation procedure where the nonlinear equation (3.10) is solved through the Newton method. In this way, we are not obliged to solve any reduced variational problem. As a consequence, we do not need to approximate the differential operator.

The operative details about the steps in the offline and online stages will be provided in the next section. Concerning hypotheses (H1)-(H3), we remark that the a priori knowledge of the equation of the shock curves (hypothesis H2) is necessary for the definition of the maps (3.4) and, consequently, of the smooth problems (3.9). On the other hand, assumptions (H1) and (H3) lead to the nonlinear equation (3.10), which represents a relevant simplification of the general Hopf-Lax formula (see [1], Thm. 2.3).

\section{A PRACTICAL APPROACH}

In this section we focus on the solution to the model problem (3.2). The following ingredients are essential:

(i) an efficient algorithm to estimate $x_{s}(\boldsymbol{\mu}, \cdot)$ in the offline and in the online stages;

(ii) for a given numerical approximation $u_{\delta}(\boldsymbol{\mu})$ of $u(\boldsymbol{\mu})$ and a numerical approximation $x_{s, \delta}(\boldsymbol{\mu})$ of $x_{s}(\boldsymbol{\mu})$, a procedure to identify the three components of the solution $u_{i}(\boldsymbol{\mu})$ for $i=1,2,3$;

(iii) a criterion to properly select the interpolation points $\left(t_{i, j}, x_{i, j}\right)$ and the bases $\left\{q_{i, j}\right\}$, for $i=1,2,3$ and $j=1, \ldots, N$.

Before dealing with these issues, we introduce the truth solver adopted to discretize the hyperbolic equation and some useful notation.

We use a finite volume conservative scheme where boundary conditions are treated through the ghost point technique $[19,20]$. Given the space-time domain $\Omega_{T,(a, b)}$, we consider the uniform space-time mesh $\left(t^{k}, x_{l}\right)$, for $k=0, \ldots, K$ and $l=1, \ldots, \mathcal{N}$, such that $x_{l}=a+(l-1) h, t^{k}=k \Delta t$ with $h=\frac{b-a}{\mathcal{N}-1}$ and $\Delta t=\frac{T}{K}$, with $\delta=(\Delta t, h)$ the space-time mesh size. For the problem at hand, the generic finite volume conservative method can be written as:

$$
u_{\delta, l}^{k+1}(\boldsymbol{\mu})=u_{\delta, l}^{k}(\boldsymbol{\mu})-\frac{\Delta t}{h}\left(F\left(u_{\delta, l-p}^{k}(\boldsymbol{\mu}), \ldots, u_{\delta, l+q}^{k}(\boldsymbol{\mu}), \boldsymbol{\mu}\right)-F\left(u_{\delta, l-p-1}^{k}(\boldsymbol{\mu}), \ldots, u_{\delta, l+q-1}^{k}(\boldsymbol{\mu}), \boldsymbol{\mu}\right)\right),
$$

where the solution $\left\{u_{\delta, l}^{k}(\boldsymbol{\mu})\right\}_{k, l}$ is an approximation to the average value of the exact solution in the $l$ th cell and at time $t^{k}$, i.e., $u_{\delta, l}^{k}(\boldsymbol{\mu}) \simeq \frac{1}{h} \int_{x_{l}-\frac{h}{2}}^{x_{l}+\frac{h}{2}} u\left(\boldsymbol{\mu}, t^{k}, x\right) \mathrm{d} x$, while $F(\boldsymbol{\mu})$ denotes the numerical flux. Throughout this work we use the Lax-Friedrichs and the Godunov monotone fluxes [19]. 


\subsection{The shock capturing algorithm}

Let us introduce the following quantities, which collect the information related to the shock:

$$
\begin{aligned}
& \Lambda(\boldsymbol{\mu}, t):=\left(t, x_{s}(\boldsymbol{\mu}, t), u_{\text {left }}(\boldsymbol{\mu}, t), u_{\text {right }}(\boldsymbol{\mu}, t)\right), \quad \Lambda(\boldsymbol{\mu}):=\left\{\Lambda(\boldsymbol{\mu}, t): t \geq t^{\star}(\boldsymbol{\mu})\right\}, \\
& \Lambda_{\delta}(\boldsymbol{\mu}, t):=\left(t, x_{s, \delta}(\boldsymbol{\mu}, t), u_{l e f t, \delta}(\boldsymbol{\mu}, t), u_{\text {right }, \delta}(\boldsymbol{\mu}, t)\right), \Lambda_{\delta}(\boldsymbol{\mu}):=\left\{\Lambda_{\delta}(\boldsymbol{\mu}, t): t \geq t_{\delta}^{\star}(\boldsymbol{\mu})\right\},
\end{aligned}
$$

with $u_{\text {left }}(\boldsymbol{\mu}, t)$ and $u_{\text {right }}(\boldsymbol{\mu}, t)$ as in Theorem 2.4. Aim of this section is to define two procedures (one for the offline stage and one for the online stage) to reconstruct $\Lambda(\boldsymbol{\mu})$. For this purpose, we subdivide each procedure into two different phases:

- the computation of $\Lambda\left(\boldsymbol{\mu}, t^{\star}(\boldsymbol{\mu})\right)$;

- the reconstruction of the whole shock $\Lambda(\boldsymbol{\mu})$.

We emphasize that these two phases characterize both the offline and the online stages.

\section{Computation of the shock starting point}

We adopt a different approach to identify the shock starting point in the offline and in the online stages. In practice, a shock is a region (here called artificial boundary layer) where the solution exhibits a strong - i.e., larger than a certain constant $H>0$ - gradient. Thus, we can reasonably assume that $x_{s}(\boldsymbol{\mu}, t)$ is located inside the high-derivative region and it exactly coincides with the maximum of the derivative. In particular, if the shock is not originated at $t=0$, we expect that the maximum of the derivative gradually grows before stabilizing at a certain large value. This remark justifies why, in the offline stage, to estimate $t^{\star}(\boldsymbol{\mu})$ we essentially check whether such a high derivative region does exists and, in this case, if the maximum of the derivative is monotonically increasing in time.

In more details, let $M_{\nabla}^{k}(\boldsymbol{\mu})$ denote the maximum of the first order discrete spatial derivative ${ }^{6}\left\{D_{l} u_{\delta}^{k}(\boldsymbol{\mu})\right\}_{l=1}^{\mathcal{N}}$ associated with the discrete values $\left\{u_{\delta, l}^{k}(\boldsymbol{\mu})\right\}_{k, l}$. The approximation for the shock starting point is thus defined as

$$
t_{\delta}^{\star}(\boldsymbol{\mu}):=k^{\star}(\boldsymbol{\mu}) \Delta t, \quad x_{s, \delta}\left(\boldsymbol{\mu}, t_{\delta}^{\star}(\boldsymbol{\mu})\right):=x_{l_{\nabla}^{\star}(\boldsymbol{\mu})}
$$

with

$$
\begin{aligned}
& k^{\star}(\boldsymbol{\mu}):=\min \left\{k \in\{0, \ldots, K\}: M_{\nabla}^{k}(\boldsymbol{\mu})>\frac{c_{\text {test }}}{N_{\text {test }}} \sum_{p=1}^{N_{\text {test }}} M_{\nabla}^{p+k}(\boldsymbol{\mu}), M_{\nabla}^{k}(\boldsymbol{\mu})>H\right\}, \\
& l_{\nabla}^{k^{\star}}(\boldsymbol{\mu}):=\arg \max _{l}\left|D_{l} u_{\delta}^{k^{\star}(\boldsymbol{\mu})}(\boldsymbol{\mu})\right|,
\end{aligned}
$$

and where $c_{\text {test }} \in(0,1)$ and $N_{\text {test }} \in \mathbb{N}$ are introduced to properly tune the oscillatory behaviour in time of the maximum of the spatial derivative. To compute $u_{\text {left }}\left(\boldsymbol{\mu}, t^{\star}(\boldsymbol{\mu})\right)$ and $u_{\text {right }}\left(\boldsymbol{\mu}, t^{\star}(\boldsymbol{\mu})\right)$, we solve the nonlinear equation (3.10), with $(t, x)=\left(t_{\delta}^{\star}(\boldsymbol{\mu}), x_{s, \delta}\left(\boldsymbol{\mu}, t_{\delta}^{\star}(\boldsymbol{\mu})\right)\right)$ via the Newton method. Since the solution to the nonlinear equation is not unique (by construction we have at least two solutions, see Prop. 3.1), we need to properly initialize the Newton scheme. The initial guesses are built by means of the values $\left\{u_{\delta, l}^{k}(\boldsymbol{\mu})\right\}_{k, l}$ as

$$
u_{l e f t, \delta}^{\text {guess }}(\boldsymbol{\mu}):=u_{\delta, l_{\nabla}^{k^{\star}}(\boldsymbol{\mu})-\Delta}^{k} \quad u_{\text {right }, \delta}^{\text {guess }}(\boldsymbol{\mu}):=u_{\delta, l_{\nabla}^{k^{\star}}(\boldsymbol{\mu})+\Delta}^{k}
$$

where $\Delta \in \mathbb{N}$ is a properly chosen index that takes into account the thickness of the artificial boundary layer.

The previous strategy is computationally too expensive for the online stage. As a consequence, at the end of the offline stage, we construct a polynomial approximation for the shock starting point and for the left- and right-solution limits by using the precomputed values for $\boldsymbol{\mu}_{j} \in \mathcal{D}$, with $j=1, \ldots, N$. In the online stage, for any new parameter $\boldsymbol{\mu} \in \mathcal{D}$, we approximate $\Lambda\left(\boldsymbol{\mu}, t^{\star}(\boldsymbol{\mu})\right)$ through the respective polynomials built offline and finally we solve (3.10) to correct the approximation of $u_{\text {left }}\left(\boldsymbol{\mu}, t^{\star}(\boldsymbol{\mu})\right)$ and $u_{\text {right }}\left(\boldsymbol{\mu}, t^{\star}(\boldsymbol{\mu})\right)$.

${ }^{6}$ In this work the discrete spatial derivative is simply computed via a centered finite difference method. We refer to [29] for further details. 


\section{Reconstruction of the entire shock curve}

Let us now turn to the second phase, where the entire shock curve (together with the left and right solution values) are recovered.

The two ingredients of the method here proposed for this purpose are:

- the Rankine Hugoniot condition (2.4);

- the characteristic equation (3.10).

Given the values $\Lambda_{\delta}\left(\boldsymbol{\mu}, t^{k}\right)$, we compute $x_{s, \delta}\left(\boldsymbol{\mu}, t^{k+1}\right)$ through an explicit discretization of the ODE (2.4) and then we evaluate $u_{\text {left }, \delta}\left(\boldsymbol{\mu}, t^{k+1}\right)$ and $u_{\text {right }, \delta}\left(\boldsymbol{\mu}, t^{k+1}\right)$ directly by solving the nonlinear equation (3.10). We observe that this method is independent of the spatial mesh size $h$ and so it can be adopted both in offline and in online stages.

Entire procedure

Algorithm 1 itemizes the whole procedure proposed for the shock capture, by distinguishing the offline from the online phases.

The proposed method essentially depends on four constants: $H>0$, used to identify the high derivative region; $\Delta$ measuring the extension of the artificial boundary layer; $c_{\text {test }}$ and $N_{\text {test }}$ characterizing the criterion to select $k^{\star}(\boldsymbol{\mu})$. A rigorous selection of these parameters is far from the purpose of this paper. We simply perform a numerical tuning of these constants during the numerical validation. We can state that the choice of $H$ and $\Delta t$ can be relatively rough: $H$ is required only to start the shock detection procedure, whereas $\Delta$ is employed simply to set the initial guesses (4.4) for the Newton algorithm. Finally, we have numerically shown that definitions (4.3) are not particularly sensitive to the values $c_{\text {test }}$ and $N_{\text {test }}$. In Algorithm 1 with notation $u=\operatorname{Newton}\left(u_{\text {start }}, t_{u}, x_{u}, \boldsymbol{\mu}_{u}\right)$ we mean that we evaluate the solution $u$ to $(3.10)$ at $(t, x, \boldsymbol{\mu})=\left(t_{u}, x_{u}, \boldsymbol{\mu}_{u}\right)$ via the Newton method, starting from the initial guess $u_{\text {start }}$.

\subsection{Smooth-jump decomposition}

We now explain how to decompose the numerical solution $u_{\delta}(\boldsymbol{\mu})$ into its three smooth components $u_{\delta}(\boldsymbol{\mu})$, for $i=1,2,3$, during the offline stage. For this purpose, we essentially exploit (3.7), i.e., the maps $\mathbf{T}_{i}(\boldsymbol{\mu})$.

Due to the intrinsic numerical viscosity of the adopted discretization scheme, we perform a pre-processing of $u_{\delta}(\boldsymbol{\mu})$ before applying (3.7). More precisely, let us consider the set of spatial indices $\left\{l_{s}^{k}\right\}_{k=0}^{K}$, given by

$$
l_{s}^{k}:=\left\{\begin{array}{l}
\arg \min _{l \in\{1, \ldots, \mathcal{N}\}}\left|\gamma_{\delta}\left(\boldsymbol{\mu}, t^{k}\right)-x_{l}\right| \quad k<k^{\star}(\boldsymbol{\mu}) \\
\arg \min _{l \in\{1, \ldots, \mathcal{N}\}}\left|x_{s, \delta}\left(\boldsymbol{\mu}, t^{k}\right)-x_{l}\right| k \geq k^{\star}(\boldsymbol{\mu}),
\end{array}\right.
$$

where $\gamma_{\delta}\left(\boldsymbol{\mu}, t^{k}\right)$ is a truth approximation of $\gamma\left(\boldsymbol{\mu}, t^{k}\right)$ defined in (3.6). We modify the solution values close to the shock so that $u_{\delta, l_{s}^{k}-1}^{k}(\boldsymbol{\mu}) \simeq u_{l e f t, \delta}\left(\boldsymbol{\mu}, t^{k}\right)$ and $u_{\delta, l_{s}^{k}}^{k}(\boldsymbol{\mu}) \simeq u_{\text {right }, \delta}\left(\boldsymbol{\mu}, t^{k}\right)$. By doing so, the effect of the numerical viscosity is significantly mitigated and possible instabilities in the empirical interpolation strategy related to the fluctuations of the smooth components close to the shock are avoided.

In addition, we introduce a computational strategy that allows us to map a function defined on a certain (space-time) partition to a different one. Let us focus on the spatial grid. We consider two couples of indices $\left(l_{a}^{1}, l_{b}^{1}\right)$ and $\left(l_{a}^{2}, l_{b}^{2}\right)$ with $1 \leq l_{a}^{p}<l_{b}^{p} \leq \mathcal{N}$ for $p=1,2$. Let $\mathbf{v}_{h} \in \mathbb{R}^{l_{b}^{1}-l_{a}^{1}+1}$ be the vector collecting the values $v_{h, l}:=\frac{1}{h} \int_{x_{l_{a}^{1}+l-\frac{3}{2}}}^{x_{l^{1}+l-\frac{1}{2}}} v(x) \mathrm{d} x$ of a real valued function $v$ defined on $\left(x_{l_{a}^{1}}, x_{l_{b}^{1}}\right)$ for $l=1, \ldots, l_{b}^{1}-l_{a}^{1}+1$. The mapping of $v$ onto the new interval $\left(x_{l_{a}^{2}}, x_{l_{b}^{2}}\right)$ leads us to introduce the new vector $\tilde{\mathbf{v}}_{h} \in \mathbb{R}^{l_{b}^{2}-l_{a}^{2}+1}$ with components

$$
\tilde{v}_{h, l}:=\frac{1}{h} \int_{x_{l_{a}^{2}+l-\frac{3}{2}}}^{x_{l_{a}^{2}+l-\frac{1}{2}}} v\left(x_{l_{a}^{1}}+\frac{x_{l_{b}^{1}}-x_{l_{a}^{1}}}{x_{l_{b}^{2}}-x_{l_{a}^{2}}}\left(y-x_{l_{a}^{2}}\right)\right) \mathrm{d} y, \quad \text { for } l=1, \ldots, l_{b}^{1}-l_{a}^{1}+1 .
$$

Throughout this paper we refer to the algorithm that approximates $\tilde{\mathbf{v}}_{h}$ for any given $\mathbf{v}_{h}$ and any two couples of indices $\left(l_{a}^{p}, l_{b}^{p}\right), p=1,2$, via the command $\tilde{\mathbf{v}}_{h}=$ FV_mapping $\left(\mathbf{v}_{h}, l_{a}^{1}, l_{b}^{1}, l_{a}^{2}, l_{b}^{2}\right)$. We approximate $(4.6)$ 


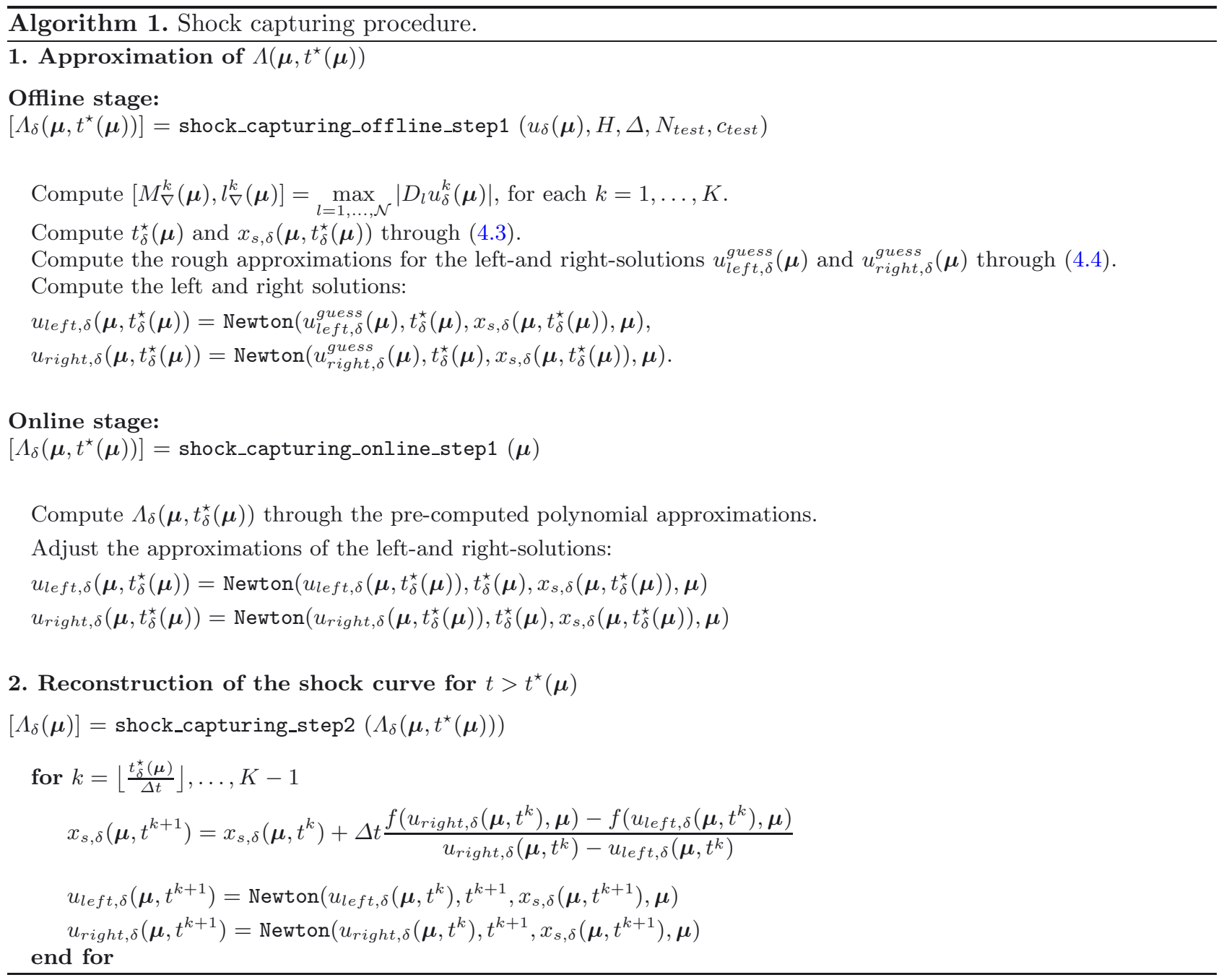

through a three point quadrature rule (see, e.g., [29]) according to which the pointwise values of $v$ are reconstructed through linear interpolation. More precisely, for each interval $\left(x_{l_{a}^{2}+l-3 / 2}, x_{l_{a}^{2}+l-1 / 2}\right)$, we approximate $v\left(x_{l_{a}^{1}}+\frac{x_{l_{b}^{1}}-x_{l_{a}^{1}}}{x_{l_{b}^{2}}-x_{l_{a}^{2}}}\left(y_{j}-x_{l_{a}^{2}}\right)\right)$ for each $y_{j}=x_{l_{a}^{2}+l-3 / 2}+h / 2(j-1), j=1,2,3$, using a piecewise linear approximation based on the values from $\left\{v_{h, l}\right\}_{l}$ (treated as pointwise evaluations) that are associated with the two closest centers of the mesh.

With obvious modifications, the same approach can be applied to the time partition.

Algorithm 2 summarizes the smooth-jump decomposition procedure.

\subsection{A greedy approach for the selection of the interpolation points}

Unlike the other algorithms of this paper, the technique we are going to present - the so-called Empirical Interpolation Method (EIM) (see, e.g., [4]) - is extremely well-known. However, at the best of our knowledge, it is the first time that such technique is applied to this framework.

Let us assume that the values $\left\{u_{\delta i, l}^{k}(\boldsymbol{\mu})\right\}_{k, l}$ for $i=1,2,3, l=1, \ldots, \mathcal{N}$ and $k=0, \ldots, K$, have already been computed for some $\boldsymbol{\mu}=\boldsymbol{\mu}_{j} \in \mathcal{D}$. Then, we can select the interpolation points $\left(t_{i, j}, x_{i, j}\right)$ and the corresponding 


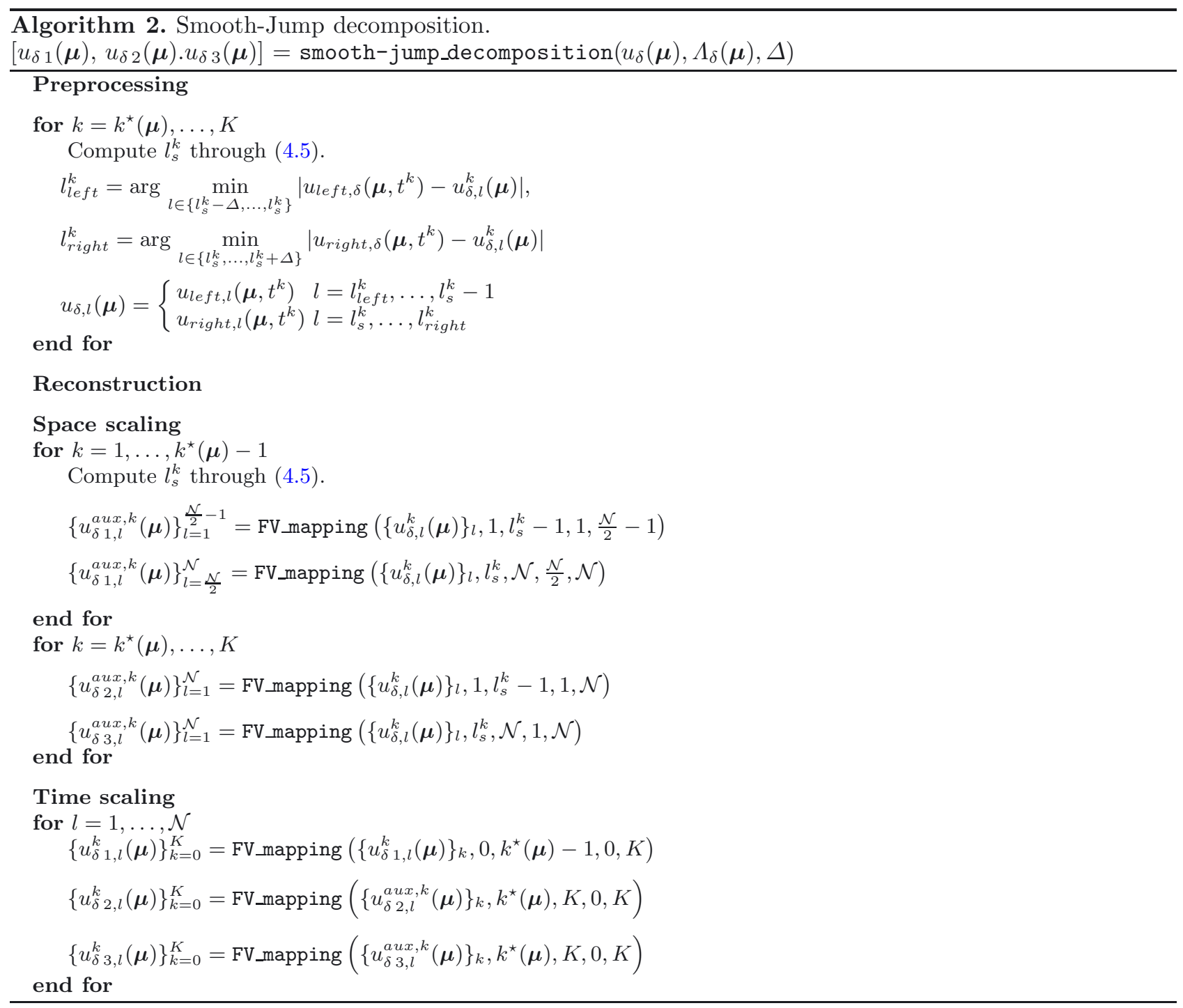

interpolatory bases $\left\{q_{i, j}\right\}$ together with the associated matrix $B_{j j^{\prime}}^{i}:=q_{i, j}\left(t_{i, j^{\prime}}, x_{i, j^{\prime}}\right)$ for $j, j^{\prime}=1, \ldots, N$ and $i=1,2,3$ via the greedy strategy proposed in [4]. Our numerical simulations show that, if the elements of the manifold exhibit regions characterized by high derivatives, the interpolation process may produce instabilities when the number of reduced bases grows. In particular, when we apply the procedure to the first component $\left\{u_{\delta 1, l}^{k}(\boldsymbol{\mu})\right\}_{k, l}$ the interpolation process might be less reliable close to the shock starting point. This is why we propose two devices to contain this drawback.

First of all, we perform a post-processing of each snapshot via a smooth filter to avoid spurious instabilities in the proximity of the regions close to the shock starting point. In more detail, we use the Matlab ${ }^{\circledR}$ tool smooth [24], which is based on a simple moving average smoothing. Then, it is convenient to prevent the greedy algorithm from selecting interpolation points associated with high solution gradients. This is justified by the fact that the approximation of the nonlinear equation (3.10) is a problem whose conditioning is good when we are far from the incoming shock, and vice versa. 


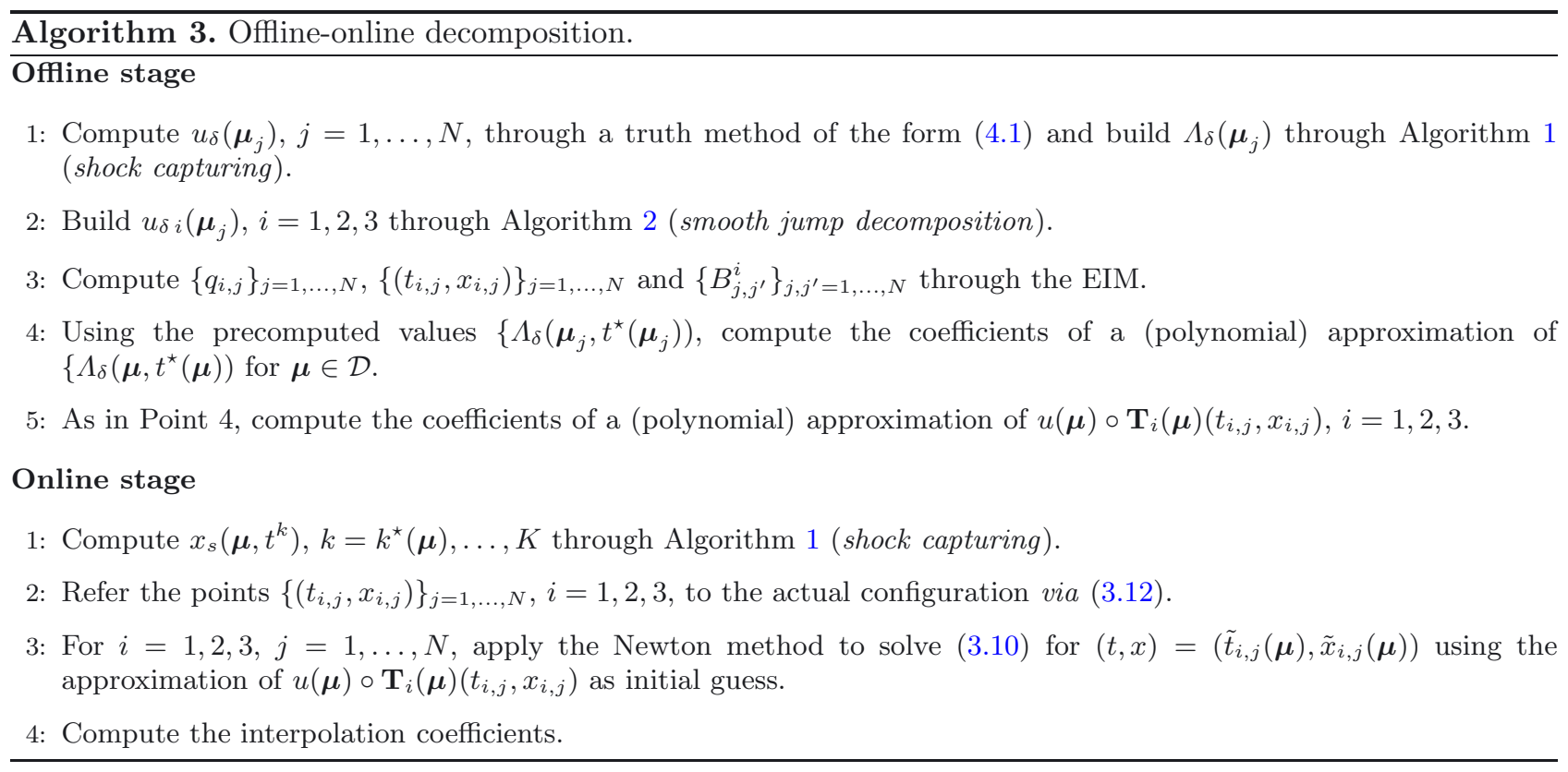

\subsection{The whole algorithm}

After detailing separately the main specific algorithms, we provide now the proposed procedure as a whole. Algorithm 3 itemizes the main steps of the global offline/online procedure. We remark that, so far, no detail has been provided about the sampling strategy adopted for the smooth problems. In the RB framework, the Greedy algorithm (see, for instance, [31]), is usually employed to properly select the parameters $\boldsymbol{\mu}_{j} \in \mathcal{D}$. This approach is based on an inexpensive and rigorous a posteriori error estimator. Nevertheless, such an estimator has not yet been developed for nonlinear hyperbolic equations. For this reason, in the numerical simulations of Section 5 we resort to equispaced $\boldsymbol{\mu}_{j}$. In Appendix A we provide a first attempt of a posteriori error indicator.

We finally observe that, in order to solve the nonlinear equation (3.10) in each mapped node (3.12) through an iterative method, it is necessary to properly select the initial guess. To do this, we employ a simple polynomial fitting. The same approach has been chosen to reconstruct $t^{\star}(\boldsymbol{\mu})$ and $x_{s}\left(\boldsymbol{\mu}, t^{\star}(\boldsymbol{\mu})\right)$. However, any other approximation technique, in principle, can be applied. Moreover, as proved in the numerical validation, while the approximations of $t^{\star}(\boldsymbol{\mu})$ and $x_{s}\left(\boldsymbol{\mu}, t^{\star}(\boldsymbol{\mu})\right)$ have to be extremely sharp, the other quantities can be roughly approximated since they are used only as initial guess for the Newton iterative algorithm.

\subsection{Input-output relationships}

The Reduced Basis method can provide a significant speed-up in the computation of input-output relationships depending on the solution of the parametrized equation.

In order to explain how to efficiently compute the input-output relation during the online stage, let us consider the following example. Let

$$
s(\boldsymbol{\mu})=\int_{\Omega_{T,(a, b)}} w(\boldsymbol{\mu}) u(\boldsymbol{\mu}) \mathrm{d} x \mathrm{~d} t
$$

be the linear-functional output of interest, where $u(\boldsymbol{\mu})$ is the solution to $(3.1), w(\boldsymbol{\mu})$ is a weight function and $\Omega_{T,(a, b)}=(0, T) \times(a, b)$. 
Our goal is to make the computation of $s(\boldsymbol{\mu})$ independent of the spatial mesh. By recalling the definition (3.7) of $u_{i}(\boldsymbol{\mu})$, for $i=1,2,3$, we have

$$
\begin{aligned}
& s(\boldsymbol{\mu})=\int_{\Omega_{T,(a, b)}} w(\boldsymbol{\mu}, t, x) u(\boldsymbol{\mu}, t, x) \mathrm{d} x \mathrm{~d} t \\
& \quad=\int_{0}^{t^{\star}(\boldsymbol{\mu})} \int_{a}^{b} w(\boldsymbol{\mu}, t, x) u(\boldsymbol{\mu}, t, x) \mathrm{d} x \mathrm{~d} t+\int_{t^{\star}(\boldsymbol{\mu})}^{T} \int_{a}^{x_{s}(\boldsymbol{\mu}, t)} w(\boldsymbol{\mu}, t, x) u(\boldsymbol{\mu}, t, x) \mathrm{d} x \mathrm{~d} t \\
& +\int_{t^{\star}((\boldsymbol{\mu}))}^{T} \int_{x_{s}(\boldsymbol{\mu}, t)}^{b} w(\boldsymbol{\mu}, t, x) u(\boldsymbol{\mu}, t, x) \mathrm{d} x \mathrm{~d} t \\
& \quad=\int_{\Omega_{T,(a, b)}} \sum_{i=1}^{3}\left(w(\boldsymbol{\mu}) \circ \mathbf{T}_{i}(\boldsymbol{\mu}) J_{i}(\boldsymbol{\mu})\right)(s, y) u_{i}(\boldsymbol{\mu}, s, y) \mathrm{d} y \mathrm{~d} s, \\
& s(\boldsymbol{\mu})=\int_{\left.\Omega_{T,\left(a, \frac{a+b}{2}\right.}\right)}\left(w(\boldsymbol{\mu}) \circ \mathbf{T}_{1}(\boldsymbol{\mu}) J_{1}(\boldsymbol{\mu})\right)(s, y) u_{1}(\boldsymbol{\mu}, s, y) \mathrm{d} y \mathrm{~d} s, \\
& +\quad \int_{\Omega_{T,\left(\frac{a+b}{2}, b\right)}\left(w(\boldsymbol{\mu}) \circ \mathbf{T}_{1}(\boldsymbol{\mu}) J_{1}(\boldsymbol{\mu})\right)(s, y) u_{1}(\boldsymbol{\mu}, s, y) \mathrm{d} y \mathrm{~d} s} \\
& +\sum_{i=2}^{3} \int_{\Omega_{T,(a, b)}}\left(w(\boldsymbol{\mu}) \circ \mathbf{T}_{i}(\boldsymbol{\mu}) J_{i}(\boldsymbol{\mu})\right)(s, y) u_{i}(\boldsymbol{\mu}, s, y) \mathrm{d} y \mathrm{~d} s,
\end{aligned}
$$

where the last equality is due to the discontinuity of $J_{i}(\boldsymbol{\mu})$ at $\left(s, \frac{a+b}{2}\right)$, for all $s \in(0, T)$. Then, by applying the EIM to each term $w(\boldsymbol{\mu}) \circ \mathbf{T}_{i}(\boldsymbol{\mu}) J_{i}(\boldsymbol{\mu})$, we obtain

$$
\begin{aligned}
\left(w(\boldsymbol{\mu}) \circ \mathbf{T}_{1}(\boldsymbol{\mu}) J_{1}(\boldsymbol{\mu})\right)(s, y) & \simeq \sum_{m=1}^{M_{1}} \Theta_{w, 1}^{m}(\boldsymbol{\mu}) w_{m}^{1}(s, y) \quad(s, y) \in \Omega_{T,\left(a, \frac{a+b}{2}\right)} \\
\left(w(\boldsymbol{\mu}) \circ \mathbf{T}_{1}(\boldsymbol{\mu}) J_{1}(\boldsymbol{\mu})\right)(s, y) & \simeq \sum_{m=1}^{M_{2}} \Theta_{w, 2}^{m}(\boldsymbol{\mu}) w_{m}^{2}(s, y) \quad(s, y) \in \Omega_{T,\left(\frac{a+b}{2}, b\right)} \\
\left(w(\boldsymbol{\mu}) \circ \mathbf{T}_{i}(\boldsymbol{\mu}) J_{i}(\boldsymbol{\mu})\right)(s, y) & \simeq \sum_{m=1}^{M_{i+1}} \Theta_{w, i+1}^{m}(\boldsymbol{\mu}) w_{m}^{i+1}(s, y)(s, y) \in \Omega_{T,(a, b)} \quad i=2,3,
\end{aligned}
$$

where $\Theta_{w, i}^{m}: \mathcal{D} \rightarrow \mathbb{R}$ and $w_{m}^{i}: \Omega_{T,(a, b)} \rightarrow \mathbb{R}, i=1,2,3,4$ and $m=1, \ldots, M_{i}$, result from the application of the EIM.

Therefore, given the RB approximations of $u_{i}(\boldsymbol{\mu}), u_{\delta i}^{R B}(\boldsymbol{\mu})=\sum_{j=1}^{N} \Theta_{u, i}^{j}(\boldsymbol{\mu}) q_{i, j}$, we can define the following parametrically affine approximation for the output (4.7)

$$
s_{R B}(\boldsymbol{\mu})=\sum_{i=1}^{2} \sum_{m=1}^{M_{i}} \sum_{j=1}^{N} \Theta_{w, i}^{m}(\boldsymbol{\mu}) \Theta_{u, 1}^{j}(\boldsymbol{\mu}) \mathcal{A}_{i j m}+\sum_{i=3}^{4} \sum_{m=1}^{M_{i}} \sum_{j=1}^{N} \Theta_{w, i}^{m}(\boldsymbol{\mu}) \Theta_{u, i-1}^{j}(\boldsymbol{\mu}) \mathcal{A}_{i j m},
$$

where

$$
\begin{aligned}
& \mathcal{A}_{1 j m}=\int_{\Omega_{T,\left(a, \frac{a+b}{2}\right)}} w_{m}^{1}(t, x) q_{1, j}(t, x) \mathrm{d} x \mathrm{~d} t \mathcal{A}_{2 j m}=\int_{\Omega_{T,\left(\frac{a+b}{2}, b\right)}} w_{m}^{2}(t, x) q_{1, j}(t, x) \mathrm{d} x \mathrm{~d} t \\
& \mathcal{A}_{3 j m}=\int_{\Omega_{T,(a, b)}} w_{m}^{3}(t, x) q_{2, j}(t, x) \mathrm{d} x \mathrm{~d} t \quad \mathcal{A}_{4 j m}=\int_{\Omega_{T,(a, b)}} w_{m}^{4}(t, x) q_{3, j}(t, x) \mathrm{d} x \mathrm{~d} t
\end{aligned}
$$

can be computed offline. 
We observe that (4.9) guarantees an efficient offline online computational decomposition: the online computation is independent of the spatial mesh whereas the temporal mesh influences the algorithm only during the shock capturing algorithm.

\section{Numerical Results}

We now assess the proposed approach on different hyperbolic problems. Three different aspects are essentially investigated. First of all, we focus only on the shock detection phase during the offline stage. Then, we validate the whole procedure detailed in Algorithm 3. Finally, we assess the input-output relationship discussed in Section 4.5.

\subsection{Shock detection and smooth just decomposition}

First of all, we check the robustness of the whole shock detection procedure used during the offline stage, i.e., of both the Algorithms 1 (offline part) and 2.

Consider the following example where the hyperbolic equation

$$
\frac{\partial}{\partial t} u(\mu)+\mu \frac{\partial}{\partial x}(u(\mu)(1-u(\mu)))=0
$$

is discretized in $\Omega_{6,(-3,3)}=(0,6) \times(-3,3)$, completed with the following initial and boundary conditions

$$
u(\mu, 0, x)=\left\{\begin{array}{lr}
\frac{1}{3} & x<0 \\
\frac{1}{3}+\frac{5}{12} x & 0 \leq x<1 \\
\frac{3}{4} & x \geq 1,
\end{array} u(\mu, t,-3)=\frac{1}{3}, \quad u(\mu, t, 3)=\frac{3}{4} .\right.
$$

for $x \in(-3,3)$ and $t \in(0,6)$, respectively. The corresponding exact solution is given by

$$
u(\mu, t, x)= \begin{cases}\frac{1}{3} & x<\min \left\{\frac{1}{3} \mu t, \frac{1}{2}-\frac{1}{12} \mu t\right\} \\ \frac{4+5 x-5 \mu t}{2(6-5 \mu t)} & \frac{1}{3} \mu t \leq x \leq 1-\frac{1}{2} \mu t, \quad t<\frac{6}{5 \mu} \\ \frac{3}{4} & x>\max \left\{1-\frac{1}{2} \mu t, \frac{1}{2}-\frac{1}{12} \mu t\right\} .\end{cases}
$$

Thus, via (3.7), the smooth components $u_{i}(\mu)$ of $u(\mu)$ are given by:

$$
\begin{aligned}
& u_{1}(\mu, s, y)= \begin{cases}\frac{1}{3} & T_{x}^{1}(s, y) \leq \frac{1}{15} s \\
\frac{4+5 T_{x}^{1}(s, y)-s}{2(6-s)} & \frac{1}{15} s \leq T_{x}^{1}(s, y) \leq 1-\frac{1}{10} s \\
\frac{3}{4} & T_{x}^{1}(s, y) \geq 1-\frac{1}{10} s\end{cases} \\
& u_{2}(\mu, s, y)=\frac{1}{3}, \quad u_{3}(\mu, s, y)=\frac{3}{4},
\end{aligned}
$$

where

$$
T_{x}^{1}(s, y):= \begin{cases}-3+\frac{7-\frac{1}{30} s}{6}(y+3) & y \leq 0 \\ \frac{1}{2}-\frac{1}{60} s+\frac{5+\frac{1}{30} s}{6} y & y \geq 0\end{cases}
$$


TABLE 1. Main quantities involved in the shock capture: comparison between approximated and exact values for different choices of the velocity.

\begin{tabular}{|l|c|c|}
\hline & $\left(t_{\delta}^{\star}(\mu), x_{s, \delta}\left(\mu, t_{\delta}^{\star}(\mu)\right), u_{\text {left }, \delta}^{\text {guess }}(\mu), u_{\text {right }, \delta}^{\text {guess }}(\mu)\right)$ & $\Lambda\left(\mu, t^{\star}(\mu)\right)$ \\
\hline$\mu=0.6$ & $(2.0260,0.4,0.3381,0.7419)$ & $\left(2, \frac{2}{5}, \frac{1}{3}, \frac{3}{4}\right)$ \\
\hline$\mu=1$ & $(1.2200,0.4,0.3383,0.7413)$ & $\left(\frac{6}{5}, \frac{2}{5}, \frac{1}{3}, \frac{3}{4}\right)$ \\
\hline$\mu=1.6$ & $(0.7620,0.4,0.3381,0.7419)$ & $\left(\frac{3}{4}, \frac{2}{5}, \frac{1}{3}, \frac{3}{4}\right)$ \\
\hline
\end{tabular}

TABLE 2. Errors in the reconstruction of the smooth components $u_{\delta 1}(\mu)$ and of the shock starting time for three different uniform space-time meshes $(\mu=1.6)$.

\begin{tabular}{|l|c|c|}
\hline & $\left\|u_{1}(\mu)-u_{\delta 1}(\mu)\right\|_{L^{2}\left(\Omega_{6,(-3,3)}\right)}$ & $\left|t_{\delta}^{\star}(\mu)-t^{\star}(\mu)\right|$ \\
\hline$\delta=\left(2 \times 10^{-3}, 10^{-2}\right)$ & $2.1 \times 10^{-2}$ & $1.2 \times 10^{-2}$ \\
\hline$\delta=\left(10^{-3}, 5 \times 10^{-3}\right)$ & $1.2 \times 10^{-2}$ & $4.0 \times 10^{-3}$ \\
\hline$\delta=\left(5 \times 10^{-4}, 2.5 \times 10^{-3}\right)$ & $7.5 \times 10^{-3}$ & $5.0 \times 10^{-4}$ \\
\hline$\delta=\left(2.5 \times 10^{-4}, 1.25 \times 10^{-3}\right)$ & $4.5 \times 10^{-3}$ & $5.0 \times 10^{-4}$ \\
\hline
\end{tabular}

Concerning the discretization scheme, we resort to the Godunov numerical flux, while we choose a uniform space-time mesh with size $\delta=\left(2 \times 10^{-3}, 10^{-2}\right)$. The parameters to be set in Algorithm 2 are chosen as

$$
H=0.3 \frac{\Delta u(\mu)}{h}, \quad \Delta=10, \quad N_{\text {test }}=20, \quad c_{\text {test }}=0.9,
$$

where $\Delta u(\mu)=\max _{(t, x)} u(\mu, t, x)-\min _{(t, x)} u(\mu, t, x)$, for $(t, x) \in \Omega_{6,(-3,3)}$.

Table 1 gathers some quantitative information for three different choices of $\mu$. In particular, we compare the approximate values for $t^{\star}(\mu), x_{s}\left(\mu, t^{\star}(\mu)\right)$, $u_{\text {left }}\left(\mu, t^{\star}(\mu)\right)$ and $u_{\text {right }}\left(\mu, t^{\star}(\mu)\right)$ (in the second column) with the corresponding exact values (in the third column). It is evident that the proposed approach is able to correctly detect the time and the starting point of the shock as well as the values of solution at the left and the right of the shock.

Let us focus now on the decomposition of the numerical solution $u_{\delta}(\mu)$ into the corresponding smooth components $u_{\delta i}(\mu)$, with $i=1,2,3$, provided by Algorithm 2. In this case, we fix the parameter $\mu$ to 1.6 and we vary the space-time discretization, by making four different choices for the space-time mesh size, i.e., $\delta=\left(2 \times 10^{-3}, 10^{-2}\right), \delta=\left(2 \times 10^{-3}, 10^{-2}\right), \delta=\left(5 \times 10^{-4}, 2.5 \times 10^{-3}\right)$ and $\delta=\left(2.5 \times 10^{-4}, 1.25 \times 10^{-3}\right)$. For each of these choices, we compute the space-time $L^{2}$-norm error associated with the approximation $u_{\delta 1}(\mu)$ as well as the error due to the approximation of the time $t^{\star}(\mu)$ via the discrete prediction $t_{\delta}^{\star}(\mu)$. The corresponding values are collected in Table 2. As expected, it turns out that the quality of the approximations provided by the proposed reconstruction algorithm depends on the selected space-time mesh. In particular, since the Godunov scheme is first order accurate, we deduce that the slight deterioration in the rate of convergence can be ascribed to the same reconstruction process.

Before concluding, we observe that, while the solution $u(\mu)$ depends on the parameter $\mu, u_{i}(\mu)$ is $\mu$-independent, for $i=1,2,3$. As a consequence, if we assume that the distance between the truth and the real solution is negligible, then the whole error associated with the offline/online strategy proposed in Algorithm 3 coincides exactly with the error related to the shock detection procedure. 


\subsection{Validation of the whole algorithm}

In this section we deal with the whole procedure, i.e., with Algorithm 3, with particular attention to the convergence of the procedure.

\subsubsection{Convergence analysis with respect to the number of basis functions}

Let us investigate the sensitivity of the convergence with respect to the selected basis of functions. To this aim, we consider the following problem;

$$
\begin{cases}\frac{\partial}{\partial t} u(\mu)+\frac{1+\mu}{6} \frac{\partial}{\partial x}\left(u(\mu)^{2}\right)=0 & (t, x) \in \Omega_{1,(-5,5)}, \\ u(\mu, 0, x)=\sin (x)+\frac{\mu}{10}\left(x^{2}-25\right) & x \in(-5,5) \\ u(\mu,-5, t)=-\sin (5) \quad u(\mu, 5, t)=\sin (5) & t \in(0,1)\end{cases}
$$

with $\mu \in \mathcal{D}=[-0.5,0.5], \Omega_{1,(-5,5)}=(0,1) \times(-5,5)$ As truth approximation, we choose the one obtained via the Lax-Friedrichs method [19] on a uniform space-time mesh.

In order to assess the convergence of the reduction procedure, we compare the reduced solution yielded by Algorithm 3 with the corresponding truth solution. When Galerkin projection is applied, we observe that the convergence is independent of the selected space-time mesh. On the contrary, in the proposed approach the space-time mesh influences the convergence of the $\mathrm{RB}$ solution with respect to the truth one. This is likely due to the fact that we use two different strategies in the offline and online stage instead of simply reducing the number of test functions as in Galerkin projection-based methods. As a consequence, the convergence of the reduced solution to the truth one is guaranteed by the convergence of the reduced solution to the exact solution. Therefore, since the difference between the exact and the truth solution depends on the space-time mesh, we have that the convergence of the reduced solution to the truth one is limited by the accuracy characterizing the truth solution as approximation of the exact one.

This is shown in Table 3 which collects, for different values of the parameter $\mu$ and for three different choices of the space-time mesh, the value of the space-time $L^{2}$-norm of the difference between the discrete reduced solution $u_{\delta}^{R B}(\mu)$ and the corresponding truth approximation $u_{\delta}(\mu)$ yielded by the discrete Lax-Friedrichs scheme. We remark that this difference decreases with a rate about equal to $\mathcal{O}(\delta)$ for large $N$ enough. Since Lax-Friedrichs method is first-order accurate, the result is in good agreement with the previous observation. The results gathered in Table 3 show that the convergence of the reduced solution to the real one is extremely fast.

\subsubsection{Convergence analysis in the presence of a shock}

Let us assume now to deal with a more complex test case. We consider the problem

$$
\frac{\partial}{\partial t} u(\mu)+\mu \frac{\partial}{\partial x}(u(\mu)(1-u(\mu)))=0(t, x) \in \Omega_{2,(-5,5)}=(0,2) \times(-5,5),
$$

where $\mu \in \mathcal{D}=[0.3,1.7]$, completed with the following initial and boundary conditions

$$
\left\{\begin{array}{l}
u(\mu, 0, x)= \begin{cases}\frac{1}{10}+\frac{1}{10} \sin (x) & -5<x<0.5 \\
\frac{1}{2}+\frac{1}{10} \sin (x) & 0.5<x<5 .\end{cases} \\
u(\mu, t,-5)=\frac{1}{10}-\frac{1}{10} \sin (5) \quad u(\mu, t, 5)=\frac{1}{2}+\frac{1}{10} \sin (5) t \in(0,2),
\end{array}\right.
$$

respectively. As truth approximation we refer to the discretization provided by the Lax-Friedrichs scheme, applied on an uniform space-time mesh. For all $\mu \in \mathcal{D}$, the solution to problem (5.6) exhibits only one shock propagating from $(0,0.5)$. Since $t^{\star}(\mu) \equiv 0$ for all $\mu \in \mathcal{D}$, we refer to $\Omega_{1}(\mu)$ and $\Omega_{2}(\mu)$ as to the two subdomains induced by the shock and, consequently, we refer to $u_{1}(\mu)$ and $u_{2}(\mu)$ as to the corresponding smooth components of the solution. Let us compare the reduced discrete solution with the truth one $u_{\delta}(\mu)$. To better understand the corresponding values gathered in Table 4 , we observe that the norm $\left\|u_{\delta}^{R B}(\mu)-u_{\delta}(\mu)\right\|_{L^{2}\left(\Omega_{2,(-5,5)}\right)}$ actually 
TABLE 3. Values of $\left\|u_{\delta}^{R B}(\mu)-u_{\delta}(\mu)\right\|_{L^{2}\left(\Omega_{1,(-5,5)}\right)}$ for different values of $N$ and $\mu$, and for three different space-time meshes: $\delta=\left(2 \times 10^{-3}, 10^{-2}\right)$ (top), $\delta=\left(10^{-3}, 5 \times 10^{-3}\right)$ (middle), $\delta=\left(5 \times 10^{-4}, 2.5 \times 10^{-3}\right)$ (bottom) .

\begin{tabular}{|l|l|l|l|l|}
\hline & $\mu=-0.35$ & $\mu=-0.15$ & $\mu=0.15$ & $\mu=0.35$ \\
\hline$\left\|u_{\delta}(\mu)\right\|_{L^{2}\left(\Omega_{1,(-5,5)}\right)}$ & 3.0807 & 2.4874 & 2.4976 & 3.0991 \\
\hline$N=2$ & $1.1 \times 10^{-1}$ & $2.2 \times 10^{-1}$ & $2.4 \times 10^{-1}$ & $1.5 \times 10^{-1}$ \\
\hline$N=4$ & $1.0 \times 10^{-2}$ & $1.1 \times 10^{-2}$ & $4.9 \times 10^{-3}$ & $4.2 \times 10^{-2}$ \\
\hline$N=8$ & $5.0 \times 10^{-3}$ & $6.9 \times 10^{-3}$ & $9.4 \times 10^{-3}$ & $1.2 \times 10^{-2}$ \\
\hline$N=16$ & $5.3 \times 10^{-3}$ & $6.6 \times 10^{-3}$ & $8.7 \times 10^{-3}$ & $1.3 \times 10^{-2}$ \\
\hline
\end{tabular}

\begin{tabular}{|l|l|l|l|l|}
\hline & $\mu=-0.35$ & $\mu=-0.15$ & $\mu=0.15$ & $\mu=0.35$ \\
\hline$\left\|u_{\delta}(\mu)\right\|_{L^{2}\left(\Omega_{1,(-5,5)}\right)}$ & 3.0807 & 2.4874 & 2.4976 & 3.0991 \\
\hline$N=2$ & $1.1 \times 10^{-1}$ & $2.2 \times 10^{-1}$ & $2.4 \times 10^{-1}$ & $1.5 \times 10^{-1}$ \\
\hline$N=4$ & $1.0 \times 10^{-2}$ & $7.1 \times 10^{-4}$ & $1.4 \times 10^{-3}$ & $3.6 \times 10^{-2}$ \\
\hline$N=8$ & $2.0 \times 10^{-3}$ & $2.5 \times 10^{-3}$ & $4.2 \times 10^{-3}$ & $4.2 \times 10^{-3}$ \\
\hline$N=16$ & $3.6 \times 10^{-3}$ & $3.3 \times 10^{-3}$ & $4.8 \times 10^{-3}$ & $5.8 \times 10^{-3}$ \\
\hline
\end{tabular}

\begin{tabular}{|l|l|l|l|l|}
\hline & $\mu=-0.35$ & $\mu=-0.15$ & $\mu=0.15$ & $\mu=0.35$ \\
\hline$\left\|u_{\delta}(\mu)\right\|_{L^{2}\left(\Omega_{1,(-5,5)}\right)}$ & 3.0807 & 2.4874 & 2.4976 & 3.0991 \\
\hline$N=2$ & $1.1 \times 10^{-1}$ & $2.2 \times 10^{-1}$ & $2.5 \times 10^{-1}$ & $1.5 \times 10^{-1}$ \\
\hline$N=4$ & $1.0 \times 10^{-2}$ & $1.0 \times 10^{-2}$ & $9.0 \times 10^{-3}$ & $3.4 \times 10^{-2}$ \\
\hline$N=8$ & $2.6 \times 10^{-3}$ & $8.3 \times 10^{-4}$ & $4.2 \times 10^{-4}$ & $1.7 \times 10^{-3}$ \\
\hline$N=16$ & $1.7 \times 10^{-3}$ & $1.8 \times 10^{-3}$ & $1.9 \times 10^{-3}$ & $2.1 \times 10^{-3}$ \\
\hline
\end{tabular}

TABLE 4. Values of the relative discrepancy between the global RB solution and the truth solution and between the corresponding smooth components for two different space-time meshes: $\delta=\left(2 \times 10^{-3}, 10^{-2}\right)($ left $), \delta=\left(10^{-3}, 5 \times 10^{-3}\right)$ (right).

\begin{tabular}{|lr|l|l|l|}
\hline & & $\mu=0.5$ & $\mu=1$ & $\mu=1.5$ \\
\hline \hline \multirow{2}{*}{$N=2$} & global & $3.14 \%$ & $3.81 \%$ & $3.46 \%$ \\
\cline { 3 - 5 } & smooth 1 & $4.16 \%$ & $6.91 \%$ & $2.84 \%$ \\
\cline { 3 - 5 } smooth 2 & $0.67 \%$ & $0.73 \%$ & $0.34 \%$ \\
\hline \hline \multirow{2}{*}{$N=4$} & $\begin{array}{l}\text { global } \\
\text { smooth 1 }\end{array}$ & $3.05 \%$ & $3.16 \%$ & $3.24 \%$ \\
\cline { 3 - 5 } & $2.28 \%$ & $1.71 \%$ & $1.48 \%$ \\
\cline { 3 - 5 } smooth 2 & $0.99 \%$ & $0.93 \%$ & $0.72 \%$ \\
\hline \hline \multirow{2}{*}{$N=8$} & global & $3.01 \%$ & $3.13 \%$ & $3.24 \%$ \\
\cline { 3 - 5 } & smooth 1 & $2.81 \%$ & $2.22 \%$ & $1.56 \%$ \\
\cline { 3 - 5 } & smooth 2 & $0.89 \%$ & $0.85 \%$ & $0.70 \%$ \\
\hline
\end{tabular}

\begin{tabular}{|lr|c|c|c|}
\hline & & $\mu=0.5$ & $\mu=1$ & $\mu=1.5$ \\
\hline \hline \multirow{2}{*}{$N=2$} & global & $2.29 \%$ & $3.09 \%$ & $2.5 \%$ \\
\cline { 3 - 5 } & smooth 1 & $4.06 \%$ & $6.98 \%$ & $2.72 \%$ \\
\cline { 3 - 5 } smooth 2 & $0.40 \%$ & $0.84 \%$ & $0.41 \%$ \\
\hline \hline \multirow{2}{*}{$N=4$} & global & $2.06 \%$ & $2.16 \%$ & $2.23 \%$ \\
\cline { 3 - 5 } & smooth 1 & $1.16 \%$ & $0.87 \%$ & $0.71 \%$ \\
\cline { 3 - 5 } & smooth 2 & $0.47 \%$ & $0.45 \%$ & $0.33 \%$ \\
\hline \hline \multirow{2}{*}{$N=8$} & global & $2.06 \%$ & $2.15 \%$ & $2.24 \%$ \\
\cline { 3 - 5 } & smooth 1 & $1.13 \%$ & $0.85 \%$ & $0.70 \%$ \\
\cline { 3 - 5 } & smooth 2 & $0.48 \%$ & $0.44 \%$ & $0.38 \%$ \\
\hline
\end{tabular}

takes into account two different contributions:

1. the one associated with the smooth jump decomposition (i.e., the distance related to the reconstruction of the shock and to the mapping). Since the RB approximation is discontinuous by construction whereas the truth solution is continuous, we expect that this component depends on the adopted mesh and on the shock equation;

2. the one related to the approximation of the smooth problems (3.9) (i.e., linked to the interpolation procedure). This component is still linked to the selected mesh (for the reasons explained in the previous paragraph) but it also depends on the RB approximation of the smooth components of the solution. 


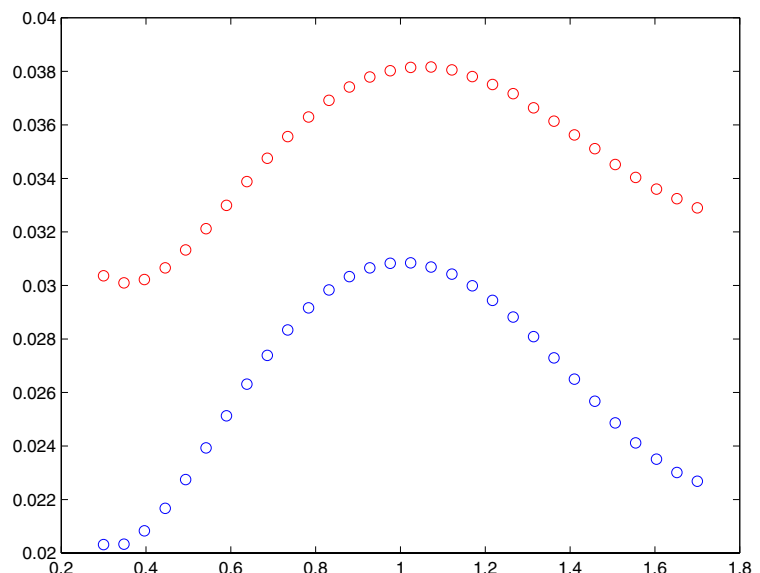

(a) $N=2$

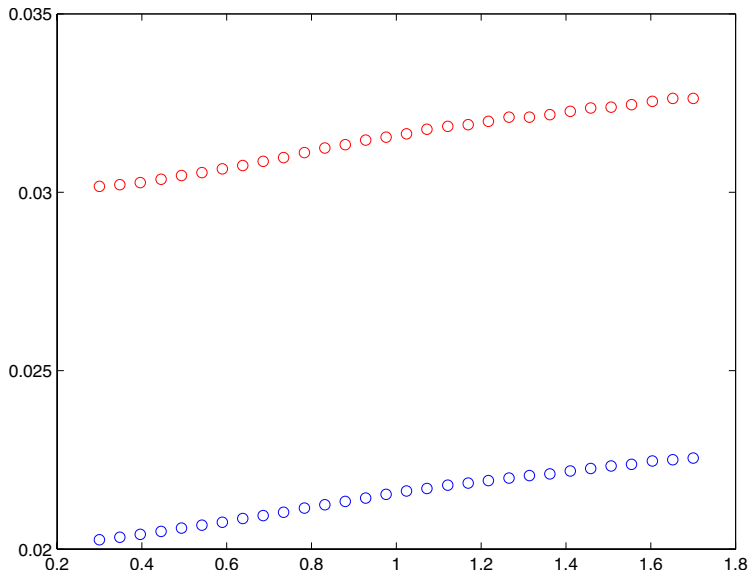

(b) $N=4$

FiguRE 3. $\left\|u_{\delta}^{R B}(\mu)-u_{\delta}(\mu)\right\|_{L^{2}\left(\Omega_{2,(-5,5)}\right)} /\left\|u_{\delta}(\mu)\right\|_{L^{2}\left(\Omega_{2,(-5,5)}\right)}$ for different values of the parameter $\mu \in \mathcal{D}$ and for two different meshes: $\delta=\left(2 \times 10^{-3}, 10^{-2}\right)$ (top: red), $\delta=\left(10^{-3}, 5 \times 10^{-3}\right)$ (bottom: blue).

To highlight the two different components, we consider both the percentage error associated with the global solution and the one associated with the smooth components. More precisely, we consider the norms $\| u_{\delta}^{R B}(\mu)-$ $u_{\delta}(\mu)\left\|_{L^{2}\left(\Omega_{2,(-5,5)}\right)} /\right\| u_{\delta}(\mu) \|_{L^{2}\left(\Omega_{2,(-5,5)}\right)}$ and $\left\|u_{\delta, i}^{R B}(\mu)-u_{\delta, i}(\mu)\right\|_{L^{2}\left(\Omega_{2,(-5,5)}\right)} /\left\|u_{\delta, i}(\mu)\right\|_{L^{2}\left(\Omega_{2,(-5,5)}\right)}, i=1,2$, for three different values of the parameter, three different bases and two different space-time meshes.

We observe that as in the previous test case, the discrepancy between the truth solution and the corresponding RB approximation, when considering the smooth components of the solution and for $N$ large enough, is approximately proportional to the mesh size. This is not true for the whole solution. This is in good agreement with the considerations at the end of Section 5.1: the contribution related to the smooth jump decomposition decreases slower than the mesh size does.

Finally, Figure 3 shows the values of $\left\|u_{\delta}^{R B}(\mu)-u_{\delta}(\mu)\right\|_{L^{2}\left(\Omega_{2,(-5,5)}\right)} /\left\|u_{\delta}(\mu)\right\|_{L^{2}\left(\Omega_{2,(-5,5)}\right)}$ for $N=2$ and $N=4$ for two different meshes. We observe that for $N=4$ the numerical error is almost uniform for all parameters, while for $N=2$ it is clearly higher in the region about $\mu=1$ : this can be explained by recalling that the RB basis has been built using the solutions for $\mu=0.3$ and $\mu=1.7$.

\subsubsection{Shock that starts at $t^{\star}(\boldsymbol{\mu})>0$}

Let us consider the following problem:

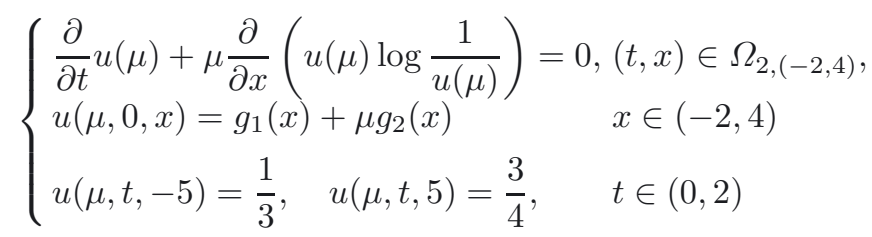

where $\Omega_{2,(-2,4)}=(0,2) \times(-2,4), \mu \in \mathcal{D}=[1.7,2.7]$ and with

$$
g_{1}(x)=\left\{\begin{array}{lrr}
\frac{1}{3} & x \leq 0 \\
\frac{1}{3}+\frac{5}{48} x^{2} & 0<x<2, \\
\frac{3}{4} & x \geq 2,
\end{array} \quad g_{2}(x)= \begin{cases}0 & x \leq-1 \\
\frac{1}{100} \sin (\pi x) & -1<x<3, \\
0 & x \geq 3 .\end{cases}\right.
$$


TABLE 5. Values of $\max _{\mu \in \Xi_{\text {train }} \subset \mathcal{D}}\left\|u_{\delta}^{R B}(\mu)-u_{\delta}(\mu)\right\|_{L^{2}\left(\Omega_{2,(-2,4)}\right)} /\left\|u_{\delta}(\mu)\right\|_{L^{2}\left(\Omega_{2,(-2,4)}\right)}$ for three different meshes $\left(\left|\Xi_{\text {train }}\right|=30\right)$.

\begin{tabular}{|l|c|c|c|}
\hline & $\delta=\left(4 \times 10^{-3}, 2 \times 10^{-2}\right)$ & $\delta=\left(2 \times 10^{-3}, 10^{-2}\right)$ & $\delta=\left(10^{-3}, 5 \times 10^{-3}\right)$ \\
\hline $\mathbf{N}=(4,2,2)$ & $2.50 \%$ & $1.49 \%$ & $1.24 \%$ \\
\hline $\mathbf{N}=(6,2,2)$ & $2.12 \%$ & $1.48 \%$ & $1.23 \%$ \\
\hline $\mathbf{N}=(4,4,4)$ & $2.22 \%$ & $1.50 \%$ & $1.15 \%$ \\
\hline
\end{tabular}

The truth approximation is based on the Godunov method on a uniform space-time mesh. For each value of the parameter, the solution to problem (5.7) exhibits only a single shock that propagates from $\left(t^{\star}(\mu), x_{s}\left(\mu, t^{\star}(\mu)\right)\right)$, where $t^{\star}(\mu)>0$.

As explained in Section 4.1, to properly initialize the shock capturing procedure it is necessary to estimate $\Lambda\left(\mu, t^{\star}(\mu)\right)$. In this simulation we consider a third order polynomial approximation for $t^{\star}(\mu), x_{s}\left(\mu, t^{\star}(\mu)\right)$, $u_{\text {left }}^{\text {guess }}\left(\mu, t^{\star}(\mu)\right)$ and $u_{\text {right }}^{\text {guess }}\left(\mu, t^{\star}(\mu)\right)$.

We evaluate the norm $\left\|u_{\delta}^{R B}(\mu)-u_{\delta}(\mu)\right\|_{L^{2}\left(\Omega_{2,(-2,4)}\right)}$ for different values of the parameter and by employing not necessarily the same number of reduced bases to approximate the three components $u_{i}(\mu)$ of the solution. In particular, with the notation $\mathbf{N}=\left(N_{1}, N_{2}, N_{3}\right)$ we mean that we employ $N_{1}, N_{2}$ and $N_{3}$ basis functions to approximate $u_{1}(\mu), u_{2}(\mu)$ and $u_{3}(\mu)$, respectively. $N_{1}$ is also the number of points used to build the polynomial approximations.

Table 5 shows the results for three different meshes. As in the previous cases, the proposed approach is able to provide rapidly convergent approximations, whereas the value of the norm $\left\|u_{\delta}^{R B}(\mu)-u_{\delta}(\mu)\right\|_{L^{2}\left(\Omega_{2,(-2,4)}\right)}$ is dominated by the contribution of the smooth jump decomposition.

Finally, Figure 4 compares the plots of the reduced and of the truth solutions for different values of the parameter $\mu$.

We observe that, despite the high gradient for $t \simeq t_{\delta}^{\star}(\mu)$, the reduced solution does not exhibits any spurious oscillations. This is likely related to the choice made for the map $\mathbf{T}_{1}(\mu)$ in (3.4) and to the approach followed to select the interpolation points.

\subsection{Input-output relationships}

We finally assess the input-output relation dealt with in Section 4.5. In particular, we choose:

$$
s(\mu)=\int_{\Omega_{1,(-5,5)}} \frac{1}{1+x^{2}} u(\mu) \mathrm{d} x \mathrm{~d} t, \quad \mu \in \mathcal{D}=[0.3,1.7],
$$

where $\Omega_{1,(-5,5)}=(0,1) \times(-5,5)$ and $u(\mu)$ is the solution to the following conservation law ${ }^{7}$

$$
\frac{\partial}{\partial t} u(\mu)+\mu \frac{\partial}{\partial x}\left(u(\mu) \log \frac{1}{u(\mu)}\right)=0, \quad(t, x) \in \Omega_{1,(-5,5)},
$$

completed with the following initial and boundary conditions:

$$
u(\mu, 0, x)=\left\{\begin{array}{l}
\frac{1}{5}+\frac{1}{10} \sin (x)-5<x<0.5 \\
\frac{1}{2}+\frac{1}{10} \sin (x) \quad 0.5<x<5,
\end{array} u(\mu, t,-5)=\frac{1}{5}-\frac{1}{10} \sin (5), \quad u(\mu, t, 5)=\frac{1}{2}+\frac{1}{10} \sin (5) .\right.
$$

To discretize this problem we resort to the Godunov method applied on a uniform space-time mesh.

In Table 6, we provide the values for $\max _{\mu \in \Xi_{\text {train }} \subset \mathcal{D}}\left\|u_{\delta}^{R B}(\mu)-u_{\delta}(\mu)\right\|_{L^{2}\left(\Omega_{2,(-2,4)}\right)} /\left\|u_{\delta}(\mu)\right\|_{L^{2}\left(\Omega_{2,(-2,4)}\right)}$ for two choices of the space-time mesh and for two different reduced bases, being $\Xi_{\text {train }}$ a discretization of the parameter domain such that $\left|\Xi_{\text {train }}\right|=30$. In Table 7 , the resulting outputs and the computational time demanded to obtain these are listed. In particular, with $N_{w}$ we denote the number of terms in the affine expansion (4.8).

\footnotetext{
7 This law is usually employed in hyperbolic traffic models. It was proposed by Greenbery and supported by experimental data from the Lincoln tunnel in New York (see [12] for further details).
} 

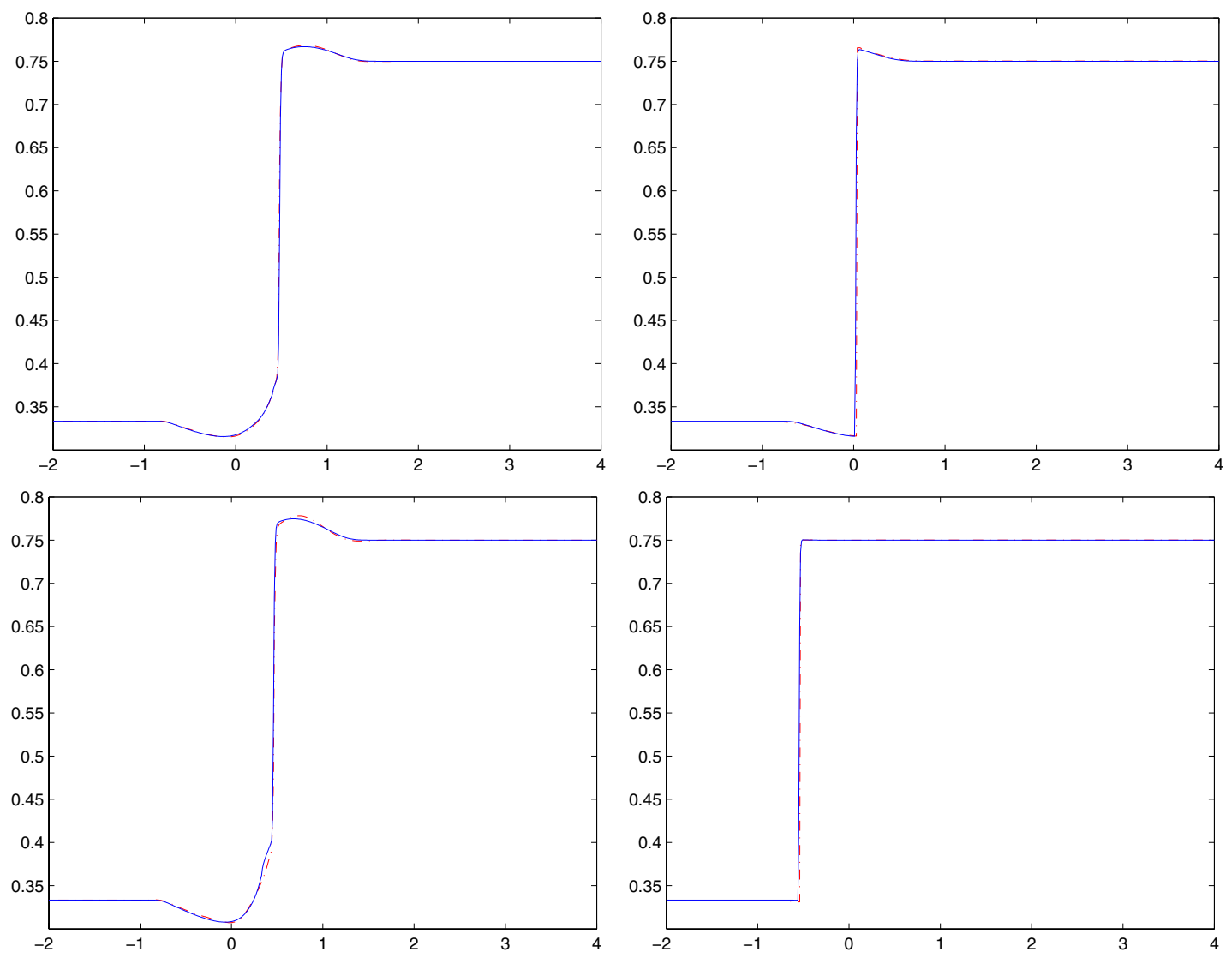

Figure 4. Reduced (dashed line) and truth (continuous line) solutions at different time steps and for different values of the parameter. $\mathbf{N}=(4,4,4)$. Solutions for $\mu=1.8$ (top) and $\mu=2.6$ (bottom) at time $t=t_{\delta}^{\star}(\mu)-\Delta t$ (left) and $t=T$ (right.)

TABLE 6. Values of $\max _{\mu \in \Xi_{\text {train }} \subset \mathcal{D}}\left\|u_{\delta}^{R B}(\mu)-u_{\delta}(\mu)\right\|_{L^{2}\left(\Omega_{2,(-2,4)}\right)} /\left\|u_{\delta}(\mu)\right\|_{L^{2}\left(\Omega_{2,(-2,4)}\right)}$ for two different meshes $\left(\left|\Xi_{\text {train }}\right|=30\right)$.

\begin{tabular}{|l|c|c|}
\hline & $\delta=\left(2 \times 10^{-3}, 10^{-2}\right)$ & $\delta=\left(10^{-3}, 5 \times 10^{-3}\right)$ \\
\hline$N=2$ & $5.60 \%$ & $5.16 \%$ \\
\hline$N=4$ & $6.00 \%$ & $5.27 \%$ \\
\hline
\end{tabular}

TABLE 7. Values of $\max _{\mu \in \Xi_{\text {train }} \subset \mathcal{D}}\left|s_{R B}(\mu)-s_{\delta}(\mu)\right| /\left|s_{\delta}(\mu)\right|$ for two different meshes and average speed-up in the output evaluation $\left(\left|\Xi_{\text {train }}\right|=30\right)$.

\begin{tabular}{|rr|c|c|}
\hline & & $\delta=\left(2 \times 10^{-3}, 10^{-2}\right)$ & $\delta=\left(10^{-3}, 5 \times 10^{-3}\right)$ \\
\hline$N_{w}=2, N_{R B}=2$ & Output error & $1.77 \%$ & $1.69 \%$ \\
\cline { 3 - 4 } & Speed-up & 170.52 & 206.31 \\
\hline$N_{w}=4, N_{R B}=2$ & Output error & $1.44 \%$ & $1.37 \%$ \\
\cline { 3 - 4 } & Speed-up & 127.63 & 145.29 \\
\hline$N_{w}=4, N_{R B}=4$ & Output error & $0.32 \%$ & $0.21 \%$ \\
\cline { 3 - 4 } & Speed-up & 102.17 & 111.82 \\
\hline
\end{tabular}


The speed-ups associated with the proposed approach are interesting. Since the truth method is an explicit scheme, the corresponding computational effort is proportional to $\mathcal{O}\left(C_{1} \mathcal{N} K\right)$, where $C_{1}$ is the cost due to the evaluation of the Godunov flux, $\mathcal{N}$ is the spatial mesh dimension and $K$ is the temporal mesh dimension. On the other hand, the computation of the reduced model is dominated by the cost associated with the shock capturing algorithm, that is $\mathcal{O}\left(C_{2} K\right)$, where $C_{2}$ is the cost demanded by the Newton algorithm to approximate the nonlinear equations (3.10).

\section{Conclusions}

In this paper we have presented a new reduced order strategy to deal with parametrized nonlinear conservation laws in the presence of shocks. For this purpose, we have essentially exploited some of the well-known analytical properties of this kind of equations, in particular the Rankine-Hugoniot condition as well as the characteristic equation.

The application of a preliminary domain partitioning and then the employment of the standard RB method on each component of the solution has already been exploited in the RB literature (see, e.g., $[17,18,21,22]$ ). On the contrary, at the best of our knowledge, the proposed interpolation strategy is new. Both the domain partitioning and the interpolation step are subject to the three hypotheses (H1)-(H3) in Section 4. The approach presented here can be extended in a straightforward way to different parametric conservation laws with more than one shock.

The numerical validation in Section 5 shows that the proposed method is able to reconstruct the solution in an efficient and reasonably reliable way. In more detail, the example in Section 5.1 proves that, despite a slight deterioration in the rate of convergence, the shock detection procedure is able to sharply reconstruct the shock equation and to compute the smooth components $u_{i}(\boldsymbol{\mu})$ of the solution, $i=1,2,3$, while the three examples in Section 5.2 show the reliability of the whole algorithm. Finally, the input-output problem discussed in Section 5.3 verifies the efficiency of the method. We highlight that the results concerning the speed-up in Table 7 are particularly meaningful since the truth numerical scheme used as benchmark is explicit.

Concerning possible future developments, we are interested in applying this approach to more general problems, in particular to quasi-linear first-order equations.

\section{ApPendix A. An A POSTERIORI ERROR INDICATOR FOR THE GREEDY SAMPLING}

In an RB framework the importance of an a posteriori error estimator is twofold. First, it is employed to reduce the online computational effort without losing the reliability of the RB approximation. Then, also the greedy sampling strategy can benefit of an inexpensive error indicator to consider larger training sets $\Xi \subset \mathcal{D}$ and to provide a better space exploration at a greatly reduced offline computational cost.

With a view to both these tasks, it is crucial that the error estimator turns out to be efficiently computable in the offline as well as in the online framework. In more detail, whereas for the online phase it is essential to have an error estimator providing a rigorous bound for the error, we are allowed to employ even non-strictly rigorous error indicators during the greedy sampling.

A posteriori error estimators for hyperbolic problems are available in the context of mesh adaptation. We recall the work by Gosse and Makridakis, (see [14]) that, starting from Kruzkov-type estimates [5], provides a result for one-dimensional scalar conservation laws discretized via E-schemes [20].

On the other hand, in the RB framework a residual a posteriori error estimator based on a lower and upper Lipschitz continuity condition has been derived in [9].

In the sequel we propose and empirically motivate a residual based error indicator, ideally suited for the greedy sampling. We first assume that the solution does not exhibit any shock. Then, we discuss how to extend the estimator to the case of discontinuous solutions by properly exploiting the decoupling strategy proposed in Section 3. Unlike the a posteriori error estimator in [9], our indicator does not rely on the underlined truth discretization and it is designed to be extended to the domain partition strategy discussed in this paper.

Throughout this appendix we omit the dependence on the parameter $\mu$ to simplify the notation. Furthermore, we settle our analysis in $\Omega_{T, \infty}=(0, T) \times \mathbb{R}$. 


\section{A.1. Error indicator for strong solutions}

We consider the two following problems:

$$
\begin{aligned}
& \begin{cases}\frac{\partial}{\partial t} u+\frac{\partial}{\partial x}(a f(u))+a_{0} u=0 & (t, x) \in \Omega_{T, \infty} \\
u(0)=u_{0} & x \in \mathbb{R},\end{cases} \\
& \begin{cases}\frac{\partial}{\partial t} u^{R B}+\frac{\partial}{\partial x}\left(a f\left(u^{R B}\right)\right)+a_{0} u^{R B}=-r_{R B}(t, x) \in \Omega_{T, \infty} \\
u^{R B}(0)=u_{0}+\bar{r}_{R B} & x \in \mathbb{R},\end{cases}
\end{aligned}
$$

with $a \in W^{1, \infty}\left(\Omega_{T, \infty}\right), a_{0} \in L^{\infty}\left(\Omega_{T, \infty}\right)$ and $u_{0} \in L^{2}(\mathbb{R})$, where $\bar{r}_{R B} \in L^{2}(\mathbb{R})$ and $r_{R B} \in L^{2}\left(\Omega_{T, \infty}\right)$. We assume that $u$ is the strong solution to problem (A.1a), while $u^{R B}$ is the strong solution to problem (A.1b). We can now introduce the error indicator

$$
\Delta^{R B}(t):=\left(\int_{0}^{t}\left\|r_{R B}(\tau)\right\|_{L^{2}(\mathbb{R})}^{2} \mathrm{~d} \tau+\left\|\bar{r}_{R B}\right\|_{L^{2}(\mathbb{R})}^{2}\right)^{\frac{1}{2}},
$$

First, we observe that the indicator is based on the strong residual, which can be computed through an offlineonline strategy: in particular, non-polynomial nonlinearities in the flux can be treated in a standard way via the EIM. Furthermore, it is possible to prove the following result, which motivates the choice made for the error indicator.

Proposition A.1. Let us assume that

$$
r_{R B} \in L^{2}\left(\Omega_{T, \infty}\right), \quad \bar{r}_{R B} \in L^{2}(\mathbb{R}), \quad f \in C^{2}(\mathbb{R}), \quad u, u^{R B} \in W^{1,1}\left(\Omega_{T, \infty}\right) .
$$

Then, the following estimate holds:

$$
\left\|u(t)-u^{R B}(t)\right\|_{L^{2}(\mathbb{R})}^{2} \leq e^{\lambda(t)}\left(\Delta^{R B}(t)\right)^{2},
$$

where $\lambda(t):=\int_{0}^{t}(1+2 \mathcal{C}(\tau)) d \tau$, with

$$
\mathcal{C}(t):=\max \left\{0,-\inf _{x \in \mathbb{R}}\left(a_{0}(t, x)+\frac{1}{2} \frac{\partial}{\partial x}\left(a f^{\prime}\left(\xi_{(t, x)}\right)\right)\right)\right\}
$$

and $\xi_{(t, x)}$ such that $f^{\prime}\left(\xi_{(t, x)}\right)=\frac{f(u(t, x))-f\left(u^{R B}(t, x)\right)}{u(t, x)-u^{R B}(t, x)}$.

The proof of this statement consists in a straightforward application of the following result from [30].

Lemma A.2. Let us consider the problem

$$
\left\{\begin{array}{l}
\frac{\partial}{\partial t} u+\frac{\partial}{\partial x}(a u)+a_{0} u=g(t, x) \in \Omega_{T, \infty} \\
u(0)=u_{0} \quad x \in \mathbb{R},
\end{array}\right.
$$

where $u_{0} \in L^{2}(\mathbb{R}), g \in L^{2}\left(\Omega_{T, \infty}\right), a \in W^{1, \infty}\left(\Omega_{T, \infty}\right)$ and $a_{0} \in L^{\infty}\left(\Omega_{T, \infty}\right)$. Then, the following estimate holds:

$$
\|u(t)\|_{L^{2}(\mathbb{R})}^{2} \leq e^{\lambda(t)}\left(\int_{0}^{t}\|g(\tau)\|_{L^{2}(\mathbb{R})}^{2} d \tau+\left\|u_{0}\right\|_{L^{2}(\mathbb{R})}^{2}\right),
$$

where $\lambda(t)=\int_{0}^{t}(1+2 \mathcal{C}(\tau)) d \tau$ and

$$
\mathcal{C}(t):=\max \left\{0,-\inf _{x \in \mathbb{R}}\left(a_{0}(t, x)+\frac{1}{2} \frac{\partial}{\partial x} a(t, x)\right)\right\} .
$$




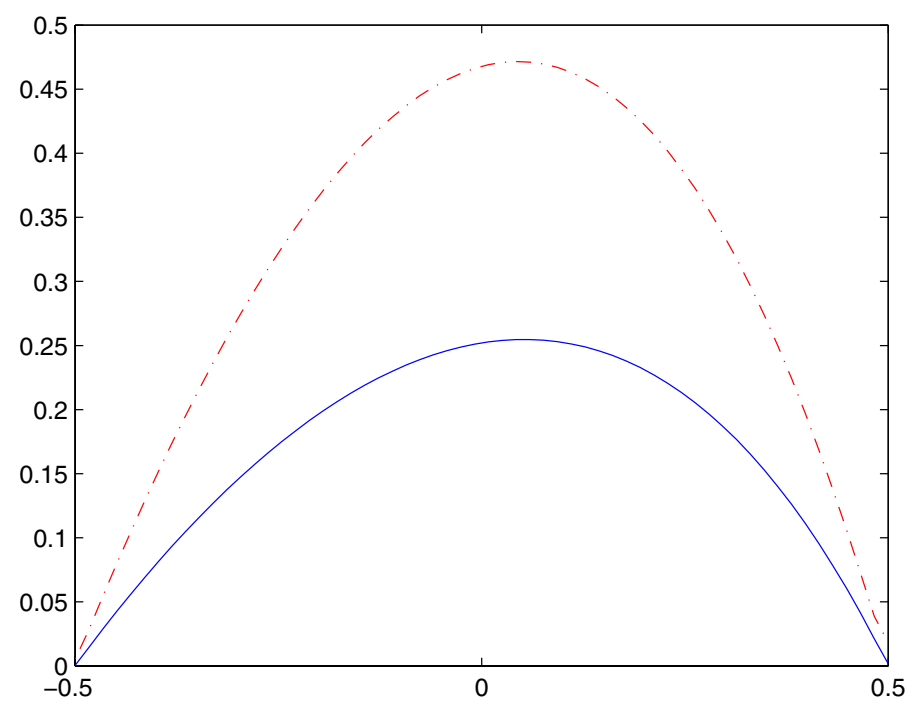

Figure 5. Comparison between the residual error indicator in (A.2) (dashed line) and the distance $\left\|u_{\delta}(\mu)-u_{\delta}^{R B}(\mu)\right\|_{L^{2}\left(\Omega_{T, \infty}\right)}$ (continuous line).

The sharpness of estimate (A.6) and consequently of (A.4) depends on the time dependent function $\mathcal{C}(t)$, which is related to the regularity of the solution. In addition, we observe that the exponential growth in time characterizing both estimates (A.6) and (A.2), which might turn to be very pessimistic in many practical cases.

We now try to numerically validate the proposed error indicator. For this purpose, we consider the same problem presented in Section 5.2.1. We identify $u_{\delta}^{R B}(\mu)$ with the reduced solution obtained by considering two equispaced snapshots. Then, we define

$$
\left\{\begin{array}{l}
\mu_{\text {max }}^{\text {truth }}:=\arg \max _{\mu \in \mathcal{D}}\left\|u_{\delta}(\mu)-u_{\delta}^{R B}(\mu)\right\|_{L^{2}\left(\Omega_{T, \infty}\right)}, \\
\mu_{\text {max }}^{\text {res }}:=\arg \max _{\mu \in \mathcal{D}} \Delta^{R B}(\mu, T) .
\end{array}\right.
$$

In particular, $\mu_{\max }^{\text {truth }}$ maximizes the distance between the reduced solution and the truth solution (computed with the Lax-Friedrichs scheme), whilst $\mu_{\max }^{r e s}$ maximizes the error indicator $\Delta^{R B}(\mu, T)$ proposed in (A.3). Figure 5 compares the error indicator with the distance $\left\|u_{\delta}(\mu)-u_{\delta}^{R B}(\mu)\right\|_{L^{2}\left(\Omega_{T, \infty}\right)}$ with respect to the parameter $\mu \in \mathcal{D}=[-0.5,0.5]$. The indicator is able to mimic the behaviour of the distance despite a certain discrepancy, more evident for $\mu \simeq \mu_{\max }^{\text {truth }}$. More quantitatively, we have that $\left|\mu_{\max }^{\text {truth }}-\mu_{\max }^{\text {res }}\right|<0.01$.

\section{A.2. Generalization to discontinuous solutions}

Let us remove now the simplifying assumption concerning the absence of shocks. We consider the model problem (3.1) on the space-time domain $\Omega_{T,(a, b)}:=(0, T) \times(a, b)$. If we assume that the error associated with the shock capturing algorithm is negligible with respect to the one introduced by the approximation of the smooth problems (3.9), we can estimate the whole error simply by taking into account the errors due to the approximation of each smooth problem (3.9).

Thus, we can write the a posteriori error indicator $\Delta^{R B}$ for the global solution as

$$
\Delta^{R B}=\sum_{i=1}^{3} \Delta^{R B, i} \sim\left\|u^{R B}-u\right\|_{L^{2}\left(\Omega_{T,(a, b)}\right)},
$$


where $\Delta^{R B, i}$ denote the a posteriori error indicator associated with the $i$ th smooth component $u^{R B, i}$ of the reduced solution, for $i=1,2,3$.

It is easy to show that the smooth problem (3.9) is equivalent to problem (A.1a) for a suitable choice of $a$ and $a_{0}$, and for $i=2,3$. On the contrary, for $i=1$ problem (3.9) is not equivalent to (A.1a), because $J_{1} \in L^{\infty}\left(\Omega_{T,(a, b)}\right) \backslash W^{1, \infty}\left(\Omega_{T,(a, b)}\right)$. A possible solution consists in considering a different map $\mathbf{T}_{1}(\boldsymbol{\mu})$. More precisely, we might require that $\mathbf{T}_{1}(\boldsymbol{\mu}): \Omega_{T,(a, b)} \rightarrow \Omega_{1}(\boldsymbol{\mu}), \mathbf{T}_{1}(\boldsymbol{\mu}) \in W^{2, \infty}\left(\Omega_{T,(a, b)} ; \mathbb{R}^{2}\right)$ and that $\mathbf{T}_{1}\left(\boldsymbol{\mu}, \frac{a+b}{2}\right)=$ $\left(t^{\star}(\boldsymbol{\mu}), x_{s}\left(\boldsymbol{\mu}, t^{\star}(\boldsymbol{\mu})\right)\right)$ for all $\boldsymbol{\mu} \in \mathcal{D}$. This approach however is not further investigated in this paper and makes the subject of a forthcoming report.

Acknowledgements. The authors are indebted with Professor Sandro Salsa for his contribution and comments on an earlier version of this work. The second author kindly acknowledges the financial support of Project PRIN 2010-2011 Innovative Methods for Water Resources under Hydro-Climatic Uncertainty Scenarios.

\section{REFERENCES}

[1] L. Ambrosio and C. de Lellis, A note on admissible solutions of 1d scalar conservation laws and 2d Hamilton-Jacobi equations. J. Hyperbol. Differ. Eq. 31 (2004) 813-826.

[2] L. Ambrosio, N. Fusco and D. Pallara, Functions of Bounded Variation and Free Discontinuity Problems. Oxford Math. Monogr. Oxford Univ. Press, Oxford (2000).

[3] C. Bardos, A.L. Roux and J. Nedélec, First order quasilinear equations with boundary conditions. Commun. Part Differ. Eq. 4 (1979) 1017-1034.

[4] M. Barrault, Y. Maday, N. Nguyen and A. Patera, An 'empirical interpolation' method: application to efficient reduced-basis discretization of partial differential equations. C. R. Math. Acad. Sci. Paris 339 (2004) 667-672.

[5] F. Bouchut and B. Perthame, Kruzkov's estimates for scalar conservation laws revisited. Trans. Amer. Math. Soc. 350 (1998) $2847-2870$.

[6] A. Bressan, Hyperbolic Systems of Conservation Laws-The One-Dimensional Cauchy Problem. Oxford Univ. Press, Oxford (2000).

[7] K. Carlberg, C. Farhat, J. Cortial and D. Amsallem, The GNAT method for nonlinear model reduction: Effective implementation and application to computational fluid dynamics and turbulent flows. J. Comput. Phys. 242 (2013) 623-647.

[8] B. Cockburn and G. Gripenberg, Continuous dependence on the nonlinearities of solutions of degenerate parabolic equations. J. Differ. Eq. 151 (1999) 231-251.

[9] M. Drohmann, B. Haasdonk and M. Ohlberger, Reduced basis approximation for nonlinear parametrized evolution equations based on empirical operator interpolation. SIAM J. Sci. Comput. 34 (2012) A937-A969.

[10] L. Evans, Partial Differential Equations. In vol. 19 of Grad. Stud. Math. 2nd edition. American Mathematical Society, Providence (2010).

[11] R. Fox and H. Miura, An approximate analysis technique for design calculations. AIAA J. 9 (1971) $177-179$.

[12] M. Garavello and B. Piccoli, Traffic Flow on Networks - Conservation Laws Models. Vol. 1. American Institute of Mathematical Sciences, New York (2006).

[13] J.F. Gerbeau and D. Lombardi, Approximated Lax Pairs for the reduced order integration of nonlinear evolution equations. Technical report, INRIA Paris-Rocquencourt (2014). Preprint ArXiv:1401.4829.

[14] L. Gosse and C. Makridakis, Two a posteriori error estimates for one-dimensional scalar conservation laws. SIAM J. Numer. Anal. 38 (2000) 964-988.

[15] M. Gunzburger, J. Peterson and J. Shadid, Reduced order modeling of time-dependent PDEs with multiple parameters in the boundary data. Comput. Methods Appl. Mech. 196 (2007) 1030-1047.

[16] B. Haasdonk and M. Ohlberger, Reduced basis method for finite volume approximations of parametrized evolution equations. Math. Model. Numer. Anal. 42 (2008) 277-302.

[17] D.B.P. Huynh, D.J. Knezevic and A.T. Patera, A static condensation reduced basis element method: approximation and a posteriori error estimation. ESAIM: M2AN 47 (2013) 213-251.

[18] L. Iapichino, A. Quarteroni and G. Rozza, A reduced basis hybrid method for the coupling of parametrized domains represented by fluidic networks. Comput. Method Appl. Mech. 221-222 (2012) 63-82.

[19] R. LeVeque, Numerical Methods for Conservation Laws. Birkhauser Verlag, Berlin (1992).

[20] R. LeVeque, Finite Volume Methods for Hyperbolic Problems. Cambridge University Press, Cambridge (2002).

[21] A. Lóvgren, Y. Maday and E. Rónquist, The reduced basis element method: application to a thermal fin problem. SIAM J. Sci. Comput. 26 (2004) 240-258.

[22] A. Lóvgren, Y. Maday and E. Rónquist, A reduced basis element method for the steady Stokes problem. ESAIM: M2AN 40 (2006) 529-552.

[23] A. Manzoni, A. Quarteroni and G. Rozza, Certified reduced basis approximation for parametrized partial differential equations and applications. J. Math. Indian 1 (2011) 1-44. 
[24] MATLAB. version 7.10.0 (R2010a). The MathWorks Inc., Natick, Massachusetts (2010).

[25] A. Noor, Recent advances in reduction methods for non-linear problems. Comput. Struct. 13 (1981) 31-44.

[26] M. Ohlberger and S. Rave, Nonlinear reduced basis approximation of parametrized evolution equations via the method of freezing. C. R. Math. Acad. Sci. Paris, Série I 348 (2013) 901-906.

[27] A. Patera and G. Rozza, Reduced Basis Approximation and a posteriori Error Estimation for Parametrized Partial Differential Equations. To appear in MIT Pappalardo Graduate Monographs in Mechanical Engineering. Massachusetts Institute of Technology (2009).

[28] T. Porsching, Estimation of the error in the reduced basis method solution of nonlinear equations. Math. Comput. 45 (1985) 487-496.

[29] A. Quarteroni, L. Sacco and F. Saleri, Numerical Mathematics. In vol. 37 of Texts Appl. Math. 1st edition. Springer (2007).

[30] A. Quarteroni and A. Valli, Numerical Approximation of Partial Differential Equations, 2nd edition. Springer-Verlag, Berlin, Heidelberg (1994).

[31] G. Rozza, D. Huynh and A. Patera, Reduced basis approximation and a posteriori error estimation for affinely parametrized elliptic coercive partial differential equations. Arch. Comput. Meth. Eng. 15 (2008) 229-275.

[32] W. Rudin, Real and Complex Analysis, 2nd edition. Mc-Graw-Hill, New York (1974).

[33] T. Bui-Thanh, M. Damodaran and K. Willcox, Proper Orthogonal Decomposition extensions for parametric applications in transonic aerodynamics. In Proc. of 16th AIAA Comput. Fluid Dynamics (2003). 